Draft Version November 8, 2018

Preprint typeset using $\mathrm{LAT}_{\mathrm{E} X}$ style emulateapj v. 04/20/08

\title{
A GEOMETRICAL HEIGHT SCALE FOR SUNSPOT PENUMBRAE
}

\author{
K. G. Puschmann ${ }^{1,2}$, B. Ruiz Cobo ${ }^{1,2}$ and V. Martínez Pillet ${ }^{1}$ \\ (1) Instituto de Astrofísica de Canarias (IAC), E-38200 La Laguna, Tenerife, Spain \\ (2) Departamento de Astrofísica, Universidad de La Laguna (ULL), E-38205 La Laguna, Tenerife, Spain \\ Draft version November 8, 2018
}

\begin{abstract}
Inversions of spectropolarimetric observations of penumbral filaments deliver the stratification of different physical quantities in an optical depth scale. However, without establishing a geometrical height scale their three-dimensional geometrical structure can not be derived. This is crucial in understanding the correct spatial variation of physical properties in the penumbral atmosphere and to provide insights into the mechanism capable of explaining the observed penumbral brightness. The aim of this work is to determine a global geometrical height scale in the penumbra by minimizing the divergence of the magnetic field vector and the deviations from static equilibrium as imposed by a force balance equation that includes pressure gradients, gravity and the Lorentz force. Optical depth models are derived from the SIR inversion of spectropolarimetric data of an active region observed with SOT on-board the Hinode satellite. We use a genetic algorithm to determine the boundary condition for the inference of geometrical heights. The retrieved geometrical height scale permits the evaluation of the Wilson depression at each pixel and the correlation of physical quantities at each height. Our results fit into the uncombed penumbral scenario, i.e., a penumbra composed of flux tubes with channelled mass flow and with a weaker and more horizontal magnetic field as compared with the background field. The ascending material is hotter and denser than their surroundings. We do not find evidence of overturning convection or field free regions in the inner penumbral area analyzed. The penumbral brightness can be explained by the energy transfer of the ascending mass carried by the Evershed flow, if the physical quantities below $z=-75 \mathrm{~km}$ are extrapolated from the results of the inversion.
\end{abstract}

Subject headings: sun: sunspot, polarization, radiative transfer, methods: numerical

\section{INTRODUCTION}

Our understanding of the structuring of the penumbral magnetic field has increased significantly over the last two decades. Three seminal works were published back in 1993. Using filter magnetograms, Title et al. (1993) found that the magnetic field inclination fluctuated by as much as \pm 18 degrees (rms) on azimuthal paths around the spot center. Lites et al. (1993), using spectropolarimetric data, identified the same fluctuations in the magnetic field, but this time in both inclination and field strength. The sense of the correlation found was that horizontal field lines tended to a have a weaker field strength. In both works, the well known Evershed flow was observed to be concentrated in the horizontal component. Simultaneously, Solanki \& Montavon (1993) proposed a model, commonly referred to as the uncombed model, of vertically interlaced magnetic field components to explain the properties of the observed net circular polarization.

The discovery of dark cored penumbral filaments by Scharmer et al. (2002), once a resolution of $\sim 0$.'1 was reached, led to the development of new models trying to explain the structure of penumbral filaments. Among them we can cite the flux pumping mechanism (Weiss et al. 2004; Brummell et al. 2008), the MISMA model (Sánchez Almeida 2005), and the "gappy" model (Spruit \& Scharmer 2006; Scharmer \& Spruit 2006). For a detailed overview we refer to recent reviews of Bellot Rubio (2009), Borrero (2009), Scharmer (2009),

Electronic address: kgp@iac.es, brc@iac.es, vmp@iac.es
Schlichenmaier (2009), and Tritschler (2009).

Many penumbral models (Schlichenmaier et al. 1998a,b; Martínez Pillet 2000; Borrero et al. 2005, 2006; Borrero 2007) share the geometry of the uncombed penumbral model: nearly horizontal magnetic flux tubes embedded in a stronger and more vertical background field. Throughout the article we will refer them as the uncombed scenario. In the frame of this scenario, several observational properties of sunspot penumbrae, like the filamentary structure, the strong Evershed outflow, and the uncombed geometry can be easily explained. Besides, it explains the properties of the observed net circular polarization (Schlichenmaier \& Collados 2002; Schlichenmaier et al. 2002; Müller et al. 2002, 2006). Recently the uncombed scenario has been modified, see Borrero \& Solanki (2010), to make it compatible with a possible existence of convective motions inside penumbral filaments as pointed out by recent observations (Bello González et al. 2005; Ichimoto et al. 2007b; Zakharov et al. 2008). One open question in the uncombed scenario is how the penumbral continuum intensity can be as large as $75 \%$ of the normal quiet Sun brightness (see, e.g., Solanki \& Rüedi 2003; Schlichenmaier \& Solanki 2003; Spruit \& Scharmer 2006). However, it is interesting to note that the simulations by Ruiz Cobo \& Bellot Rubio (2008) can explain up to $50 \%$ of the quiet Sun intensity if the Evershed flow consists of horizontal flow channels with a length of several $\mathrm{Mm}$ that bring hot material from deeper layers. Indeed, the problem of the penumbral heating was a strong motivation for Scharmer \& Spruit 
(2006) and Spruit \& Scharmer (2006) to propose the gappy penumbral model, in which field-free overturning convection occurs inside the penumbra and efficiently heats its atmosphere. However, this model has not yet been confronted to spectropolarimetric observations. In particular, it is unclear if this model is able to reproduce the observed net circular polarization. However, for that to happen, the magnetic field inside the filaments should be around $1000 \mathrm{G}$, which seems to be incompatible with the concept of a field-free gap (Borrero \& Solanki 2010). Recently, Scharmer (2008) proposed the possibility of strongly reduced (although non-zero) magnetic field inside the field-free gap. These modifications of both scenarios, uncombed (including convection inside flux tubes) and gappy (including significant magnetic field inside the field-free gaps), could in the end produce similar observational effects, although they are based on different physical mechanisms.

Simulations based on radiative magneto-convection in inclined magnetic fields can help to shed light on open questions. First attempts to simulate penumbral structure in small slab-like sections of sunspots (Heinemann et al. 2007; Rempel et al. 2009a) resulted in rather narrow penumbral regions. Recent numerical simulations by Rempel et al. (2009b) seem to reproduce many observational properties of penumbral filaments: e.g. the filamentary structure of the penumbra, the Evershed flow, some supersonic velocities (although the observed ones are certainly more complex, see e.g. Bellot Rubio et al. 2004; Ichimoto et al. 2007a; Martínez Pillet et al. 2009), the correlation between inclination and strength of the magnetic field, and the penumbral brightness. Nevertheless, the resulting penumbra is still somewhat immature, revealing too short and too fragmented filaments. Dark lanes along bright filaments observationally found by Scharmer et al. (2002) form only occasionally. At present, these simulations do not allow a direct comparison with spectropolarimetric observations, since up to our knowledge no synthesized spectra are available. Surprisingly, the simulated penumbrae share some properties with both the uncombed and the gappy scenarios: i.e. convective motions inside zones harboring nearly horizontal and relatively strong (around $1 \mathrm{kG}$ ) magnetic fields. The vertical slices across a penumbral filament taken from a snapshot of Rempel et al's simulations and presented in Figure 1 of Borrero (2009) point to the co-existence of significant magnetic fields and overturning velocities.

Observationally, 3D visualizations of penumbral filaments can only be obtained from inversions of spectropolarimetric data (in the absence of robust results from local helioseismology, Gizon et al. 2009). While Milne-Eddington inversions only yield the physical parameters at an average optical depth (Sánchez Almeida et al.|1996), more sophisticated inversion codes like SIR (Ruiz Cobo \& del Toro Iniesta 1992) or SPINOR (Frutiger et al. 2000) provide the stratification of physical parameters in an optical depth scale. Several 3D optical depth models have been published in the recent past (see, e.g., Westendorp Plaza et al. 2001a, b; Mathew et al. 2003; Sánchez Cuberes et al. 2005; Beck 2006, 2008).

The highest spatial resolution input data for this endeavor are currently provided by the CRisp Imag- ing SpectroPolarimeter (CRISP, Scharmer 2006) and by the Hinode/SP spectropolarimeter (Lites et al. 2001, see also Tsuneta et al. 2008). In the near future the Gregor Fabry Perot interferometer (GFPI, see e.g. Puschmann et al. 2006, 2007) and its full Stokes polarimeter (Bello González \& Kneer 2008; Balthasar et al. 2009) may reveal spectropolarimetric data at even higher spatial resolution once Gregor (Volkmer et al. 2007) is in operation. The ground based $2 \mathrm{D}$ observations take advantage of image reconstruction techniques (see e.g. Löfdahl 2002; van Noort et al. 2005; Puschmann \& Sailer 2006; Denker et al. 2007; Bello González \& Kneer 2008; Wöger \& von der Lühe 2008) to improve spatial resolution and to reduce spatial crosstalk from variable seeing.

Jurcák et al. (2007) and Jurcák \& Bellot Rubio (2008) have carried out inversions of Hinode data and found an atmosphere that is basically consistent with the uncombed scenario: horizontal channels carrying the Evershed flow are substantially magnetized. These channels appear at large optical depths, but no information about geometrical heights is obtained.

Given the highly fluctuating densities that are expected in the penumbra, the interpretation of an optical depth scale as a good approach for geometrical heights can be misleading and a reliable transformation to geometrical heights is mandatory. Besides, to establish a geometric height scale is important for the determination of the electrical current vector $\vec{J}$ that is crucial for the determination of ohmic energy dissipation, and for a test of the reliability of $a b$ initio MHD simulations. While traditionally in quiet Sun the transformation from an optical depth scale to a geometric one has been done by assuming hydrostatic equilibrium (see, for example, Puschmann et al. 2005), this is not justified in the magnetized penumbra. The force balance in this case must include magnetic forces which require the calculation of horizontal and vertical spatial derivatives of the magnetic field.

An absolute geometrical height scale for penumbrae has been derived by Sánchez Almeida (2005) from a spectropolarimetric inversion under the MISMA hypothesis by imposing equal total pressure between adjacent pixels, although neglecting the magnetic tension in the Lorentz force. Carroll \& Kopf (2008) obtained the stratification of physical quantities in geometrical height applying a neural network inversion technique based on MHD simulations of quiet Sun. The application of this technique on sunspot penumbrae might be straight forward, using e.g. the simulations of Rempel et al. (2009b) as input.

The main objective of the work presented here is to infer a 3D geometrical model of the penumbra by imposing the solenoidality of the magnetic field and the dynamic equilibrium including the Lorentz force. This model allows the determination of the Wilson depression at each pixel, the correlation of physical quantities at each height, and the evaluation of the energy budget transferred by mass motions.

\section{OBSERVATIONS}

The active region AR 10953 near solar disk center was observed using the spectropolarimeter (SP) of the Solar Optical Telescope (SOT) on-board the Hinode spacecraft 


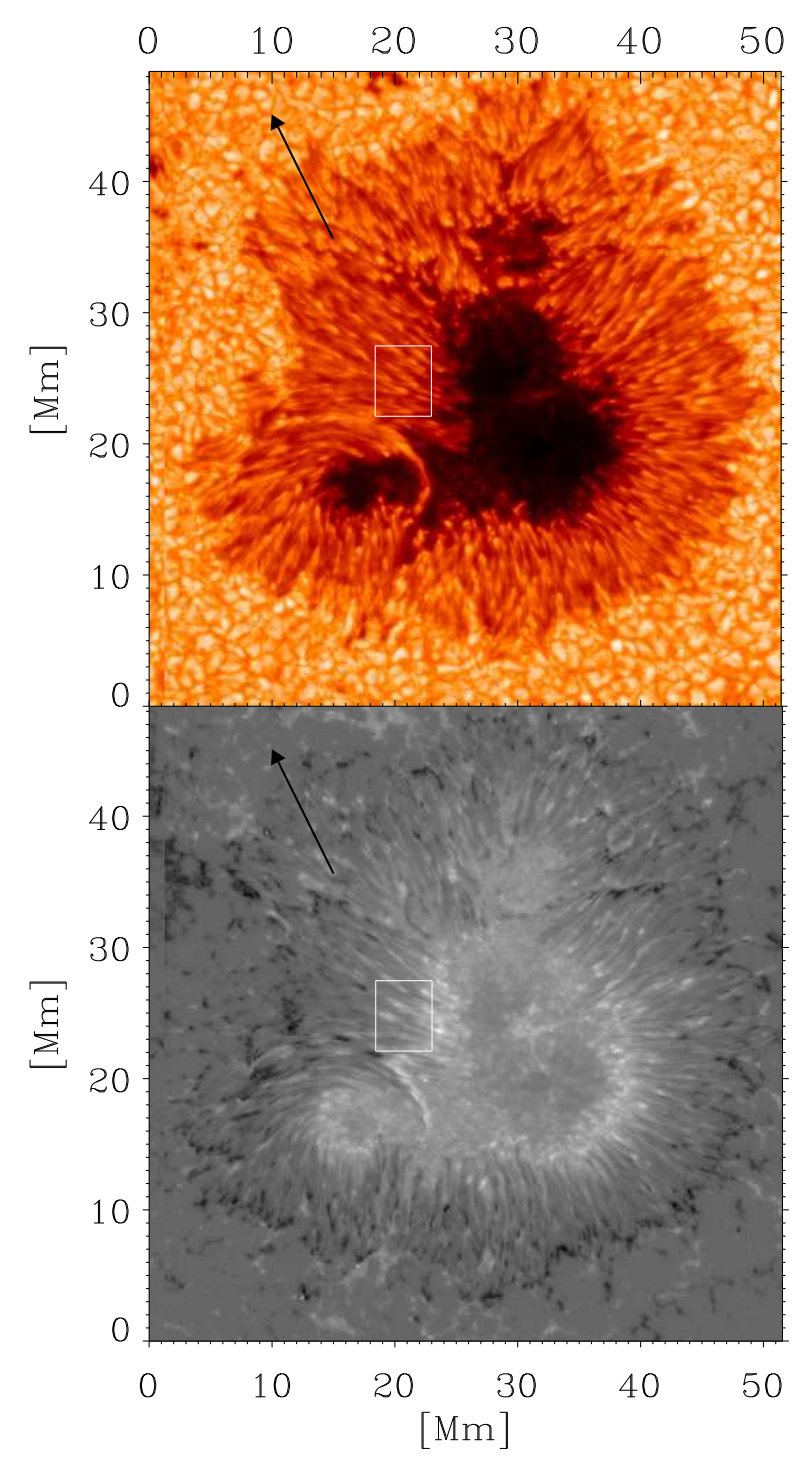

FIG. 1. - Upper panel: Continuum intensity at $630.2 \mathrm{~nm}$ of a fragment of the active region AR 10953 observed by SOT on-board of the Hinode spacecraft. The white rectangle marks the area under study in the present paper. The arrow indicates the direction to disk center. The slit is vertically oriented. Bottom panel: Stokes $\mathrm{V}$ at $+286 \mathrm{~m} \AA$ from the line center.

on the $1^{\text {st }}$ of May 2007, between 10:46 and 12:25 UT. The region was scanned with a step size of $00^{\prime \prime} 148$ and a slit width corresponding to $0^{\prime \prime} 158$, recording the full Stokes vector of the pair of the neutral iron lines at $630 \mathrm{~nm}$ with a spectral sampling of $21.53 \mathrm{~m} \AA$. The spatial resolution was about $0^{\prime \prime} 32$.

The integration time was $4.8 \mathrm{~s}$, resulting in a noise level of approximately $1.2 \times 10^{-3}$. The wavelength calibration was done assuming that the average umbral profile does not exhibit velocities.

For reasons of simplicity this article centers on a section of the inner penumbra of the large sunspot presented in Figure 1. The analyzed area is marked by a white rectangle centered at an heliocentric angle $\theta=4.63^{\circ}$. The arrow indicates the direction to disk center. In figure coordinates, the disk center would be at $X=-5.5 \mathrm{Mm}$ and
$Y=74 \mathrm{Mm}$. This area has been chosen because it consists of homogeneous and radial aligned filaments. In the bottom panel of Figure 1 we show the map of Stokes V at $+286 \mathrm{~m} \AA$ from the line center. In this map, the black points correspond to pixels in which the magnetic field shows a reverse polarity and harbors strong downflows (Ichimoto et al. 2007a). The area analyzed in this paper does not include such points. Nevertheless, provided that these strong downflows are mainly located in the mid and outer penumbra, the results of this paper can be considered representative for the inner penumbra. In a posterior paper we will apply the method described in this article to the entire sunspot.

\section{INVERSION PROCEDURE}

To derive the physical parameters of the solar atmosphere as a function of continuum optical depth, i.e. temperature $T(\tau)$, magnetic field strength $B(\tau)$, field inclination $\gamma(\tau)$, field azimuth $\phi(\tau)$, and line of sight velocity $v_{\text {los }}(\tau)$, the SIR inversion code (Ruiz Cobo \& del Toro Iniesta 1992) was applied on the spectropolarimetric data set. In the region of the inner center-side penumbra under study, all Stokes profiles are slightly asymmetric, are not multi-lobed and do not show strong displacements. Consequently, there is not sufficient information to obtain a two-component atmosphere unambiguously. Therefore, we use an one-component inversion to obtain the stratification of plasma parameters in each pixel. The same procedure has already been applied on sunspot penumbral data observed with the Hinode/SP by e.g. Jurcák \& Bellot Rubio (2008). The spectropolarimeter on-board Hinode allows us to distinguish the fine structure of the penumbra with a resolution of $00^{\prime \prime} 3$. The dark cored penumbral filaments discovered by Scharmer et al. (2002), with a typical size less than $0{ }^{\prime \prime} 1$, could indicate the existence of smaller physical structures, but could also be generated by temperature differences present in iso-tau layers above large structures like flux tubes (Ruiz Cobo \& Bellot Rubio 2008) or field-free gaps (Spruit \& Scharmer 2006). In any case, our results have to be interpreted as the averaged properties of structures on a scale of 0.3 .

The free physical parameters are calculated by SIR at different optical depth points (nodes). We have chosen 5 nodes in $T(\tau), 3$ nodes in $B(\tau)$ and $v_{\text {los }}(\tau)$, and 2 nodes in $\gamma(\tau)$ and $\phi(\tau)$. The final temperature stratification is obtained by spline interpolation. We have used a parabolic interpolation for $B(\tau)$ and $v_{\mathrm{los}}(\tau)$ and linear interpolation in case of $\gamma(\tau)$ and $\phi(\tau)$. We have neither considered microturbulent velocities nor stray light contamination. The final synthesized profiles have been convolved with a macroturbulent velocity $v_{\text {mac }}$ as an additional free parameter of the inversion.

In Figure 2, we present the results of the inversion at an arbitrarily chosen pixel. Note that the synthesized spectral lines even reproduce the asymmetries present in the observational Stokes profiles (left four panels in Figure 21). In the right four panels of this figure, we present the stratification of $T, v_{\text {los }}, B, \gamma$, and $\phi$ versus the continuum optical depth. The error bars calculated at the different nodes are also shown. The sunspot has negative polarity $\left(\gamma>90^{\circ}\right)$. We do not perform any correction of the azimuth to solve the $180^{\circ}$ ambiguity, since all resulting azimuth values are in the range between $-90^{\circ}$ and 

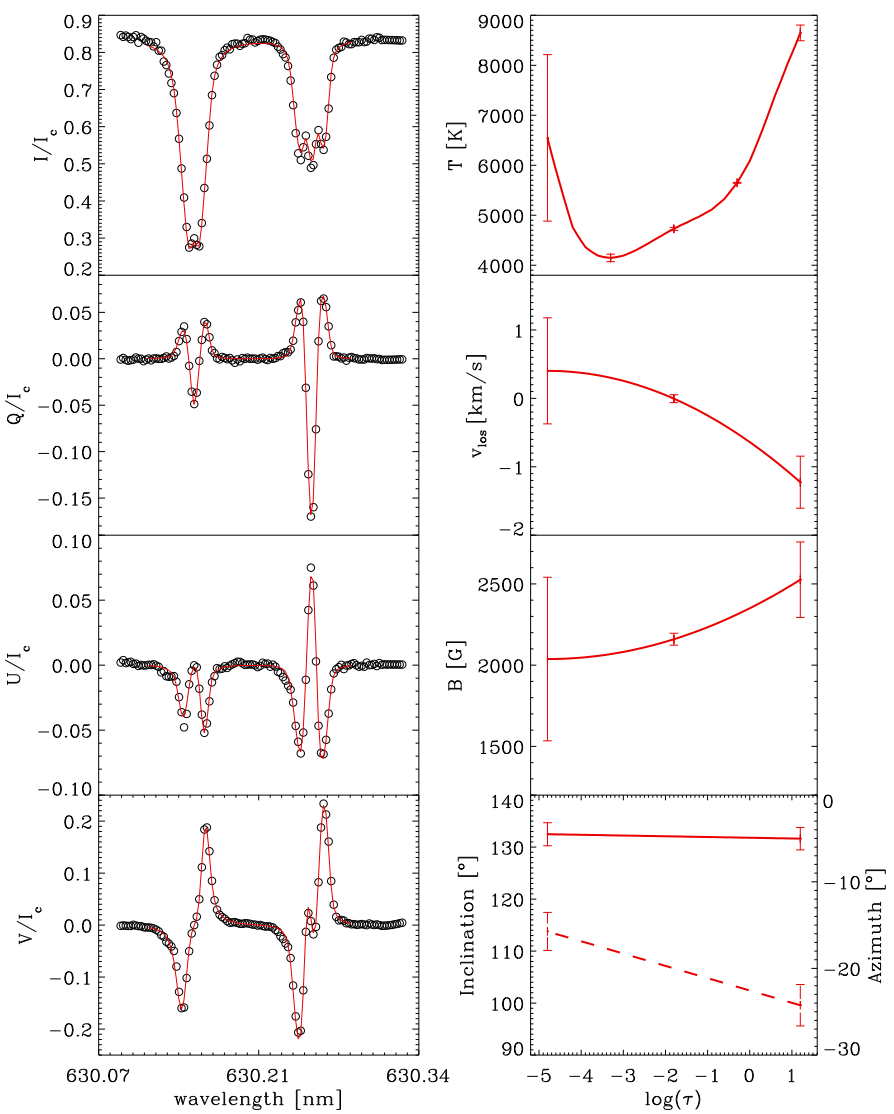

FIG. 2.- Left column: Normalized observed Stokes $I, Q, U$ and $V$ profiles of one pixel (circles); The red solid lines correspond to the best fits of synthesized profiles. Right column: Corresponding stratification of temperature $T$, line-of-sight velocity $v_{\text {los }}$, magnetic field strength $B$, field inclination $\gamma$ and field azimuth $\phi$ (dashed line) vs. logarithm of continuum optical depth at $500 \mathrm{~nm} \log (\tau)$; over-plotted are the error bars calculated at the different nodes.

$90^{\circ}$ showing a smooth spatial variation across the field of view $(\mathrm{FOV})$.

\section{DETERMINATION OF A GEOMETRICAL HEIGHT SCALE}

The inversion method delivers for each pixel the stratification of an atmospheric model vs. continuum optical depth, i.e. we obtain $\vec{B}(x, y, \tau), T(x, y, \tau)$, etc... (see Figure 3). Taking into account that the region under investigation is not exactly placed at disk center $(\mu=0.997)$, our $X Y$-plane is not parallel to the local solar surface. A transformation of the reference system can only be applied a posteriori, once the $z$-scale has been obtained. Besides, it is impossible to perform this transformation without the knowledge of horizontal velocities. Consequently, we consider the $z$-axis to be parallel to the line-of-sight direction throughout this article. We will term the $z$-axis as the "vertical" and accordingly the perpendicular plane as the "horizontal" plane.

A geometrical height scale $z(x, y, \tau)$ can be obtained by integrating

$$
d z=-\frac{1}{\kappa \rho} d \tau .
$$
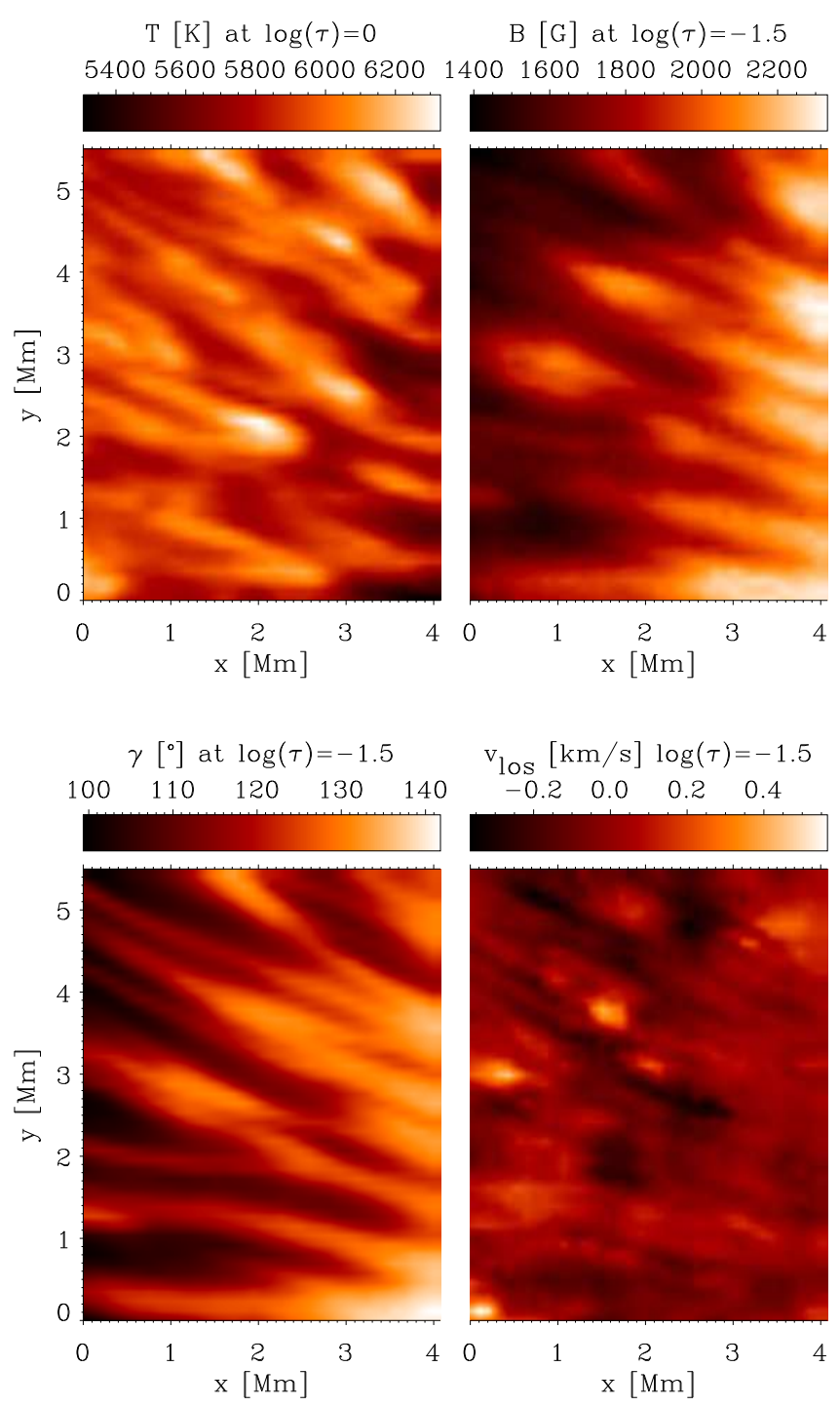

FIG. 3.- Maps of temperature $T$ at $\log (\tau)=0$, and maps of magnetic field strength $B$, field inclination $\gamma$, and line of sight velocity $v_{\operatorname{los}}$ at $\log (\tau)=-1.5$. Negative and positive velocities correspond to upflows and downflows, respectively.

For the integration of equation (1), three ingredients are needed: $\kappa$ (continuum absorption coefficient per gram), $\rho$ (mass density) and the boundary condition $Z_{\mathrm{W}}=-z(\tau=1)(\text { Wilson depression })^{1}$. The first ingredient, $\kappa(x, y, \tau)$, is evaluated by $\operatorname{SIR}$ from $T(x, y, \tau)$, the gas pressure $\left(P_{\mathrm{g}}\right)$ and the abundances. However, as we will see below, $\kappa$ is nearly independent of $P_{\mathrm{g}}$. The stratification of $P_{\mathrm{g}}$ and $\kappa$ are obtained under the assumption of hydrostatic equilibrium (HE), i.e., by the integration of

$$
d P_{\mathrm{g}} / d \tau=g / \kappa,
$$

where $g$ represents gravity. In each step of the integration (i.e. at each $\tau$ ) $\kappa$ and $P_{\mathrm{g}}$ are found iteratively such that both variables are compatible with the ioniza-

${ }^{1}$ We introduce a change of sign to be consistent with the usual definition of the Wilson depression, i.e. positive values for depressed $\tau=1$ layers. 
tion equilibrium in LTE (Saha equation). For the gas pressure boundary condition at the top layer $(\log \tau=-$ 4.8) we used $655 \mathrm{dyn} / \mathrm{cm}^{2}$ (taken from Vernazza et al. 1981). Solving the HE (equation (2)) with boundary conditions differing by two orders of magnitude $\left(P_{\mathrm{g}}(\log \tau=-\right.$ $4.8)=10 \mathrm{dyn} / \mathrm{cm}^{2}$ and $\left.P_{\mathrm{g}}(\log \tau=-4.8)=1000 \mathrm{dyn} / \mathrm{cm}^{2}\right)$ the resulting stratification of the absorption coefficients $\left(\kappa_{10}(\tau)\right.$ and $\left.\kappa_{1000}(\tau)\right)$ is practically invariant. The ratio between $\kappa_{1000}(\tau)$ and $\kappa_{10}(\tau)$ approximately follows the empirical law:

$$
\frac{\kappa_{1000}(\tau)}{\kappa_{10}(\tau)} \sim 1-\frac{5 \cdot 10^{-5}}{\tau},
$$

i.e. at $\log \tau>-2$ the difference is smaller than $0.5 \%$.

The pressure stratification is used for the calculation of the second ingredient, the density, using the equation of state for an ideal gas considering partial ionization.

By choosing an arbitrary Wilson depression $Z_{\mathrm{W}}$ at all pixels, a geometric height scale $z(x, y, \tau)$ can be constructed after integration of equation (11) (which would be a HE height scale). $\vec{B}(x, y, z)$ maps are then obtained by the interpolation of the $\vec{B}(x, y, \tau)$ maps resulting from the SIR inversion. Such $\vec{B}(x, y, z)$ maps have a divergence different from zero. Furthermore, this model would not be in mechanical equilibrium. An optimum choice of $Z_{\mathrm{W}}(x, y)$ should minimize at a given height both the divergence of the magnetic field $\nabla \cdot \vec{B}$ and the error in the equation of motion.

Neglecting viscosity, the equation of motion can be written as

$$
\rho \frac{d \vec{v}}{d t}=\vec{J} \times \vec{B}+\rho \vec{g}-\nabla P_{g},
$$

where $\vec{v}$ stands for velocity and $\vec{J}$ for the current density. The acceleration on the left hand side of equation (4) can be decomposed in a temporal derivative and an advective term

$$
\frac{d \vec{v}}{d t}=\frac{\partial \vec{v}}{\partial t}+(\vec{v} \cdot \nabla) \vec{v} .
$$

Due to the extended lifetime of penumbral filaments of $\sim 1$ hour (see e.g. Sütterlin et al. 2004; Langhans et al. 2007) compared to the sound travel time across a filament, their velocity can be considered stationary and we thus neglect the partial temporal derivative $\frac{\partial \vec{v}}{\partial t}$ in equation (55). Since the photospheric magnetic field is nearly frozen into the plasma, we can assume, as an order of magnitude estimation, that the material moves along the field lines and derive $\vec{v}$ from $v_{\mathrm{z}}=-v_{\mathrm{los}}, \gamma$, and $\phi$. Note that positive $v_{\text {los }}$ corresponds to a red-shift while positive $v_{\mathrm{z}}$ are upflows.

We also negelect the dynamic term $(\vec{v} \cdot \nabla) \vec{v}$, since it can be shown to be much smaller than the advective term. In Figure 4 we plot the histogram of the errors introduced by neglecting the dynamic term: ratio $_{x}$, ratio $y$, $\mathrm{ratio}_{\mathrm{z}}$, which represent the ratio between the advective term (the second term on the right hand side of equation (5)) and the dynamic term (second and third terms on the right hand side of equation (44) without considering the Lorentz force for each of the three components:

$$
\operatorname{ratio}_{\mathrm{x}}=-\rho \frac{(\vec{v} \cdot \nabla) v_{\mathrm{x}}}{\partial P_{g} / \partial x}
$$

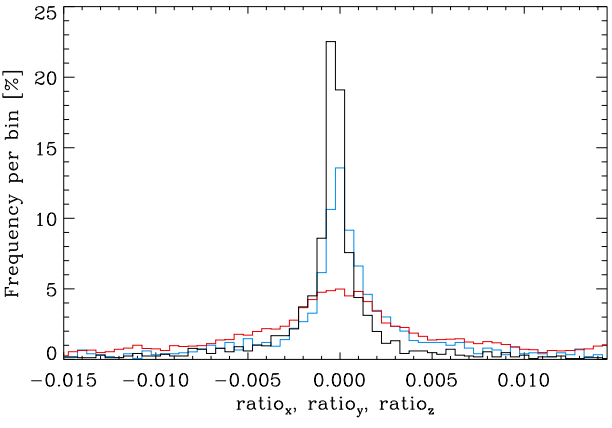

FIG. 4.- Histograms of the ratio between the advective term and the dynamic term without considering the Lorentz force for each of the three components evaluated at a height of $200 \mathrm{~km}$ in the penumbral region presented in Figure 1 ratio (black), ratioy (red), ratio $_{z}$ (blue). Binsize of $5 \times 10^{-4}$.

$$
\begin{aligned}
\text { ratio }_{\mathrm{y}} & =-\rho \frac{(\vec{v} \cdot \nabla) v_{\mathrm{y}}}{\partial P_{g} / \partial y} \\
\operatorname{ratio}_{\mathrm{z}} & =-\rho \frac{(\vec{v} \cdot \nabla) v_{\mathrm{z}}}{\partial P_{g} / \partial z+\rho g} .
\end{aligned}
$$

The magnitudes of equation (6) have been evaluated at the reference height of $200 \mathrm{~km}$ where the minimization procedure will be applied. The neglection of the advective term is justified since most of the values are below $1 \%$.

Let us define then the residual force $\vec{F}$ to be

$$
\vec{F}=\vec{J} \times \vec{B}+\rho \vec{g}-\nabla P_{g},
$$

with $P_{g}$ inferred from equation (2). In our approximation $\vec{F}$ is zero if the equation of motion is fulfilled. To ensure the physical meaningfulness of the solution also $\nabla \cdot \vec{B}$ must be zero.

We define a merit function as

$$
\chi^{2}=\sum_{\text {pixels }} w_{1}\left(F_{\mathrm{x}}^{2}+F_{\mathrm{y}}^{2}+F_{\mathrm{z}}^{2}\right)+w_{2}(\nabla \cdot \vec{B})^{2} .
$$

where $w_{1}, w_{2}$ are coefficients introduced to adequately weight the contribution of both $\nabla \cdot \vec{B}$ and $\vec{F}$. By introducing vertical displacements of the atmospheric models at each pixel, we minimize the merit function of equation (8). Therefore, we used a genetic algorithm that iteratively changes an array $D_{\mathrm{z}}(x, y)$ containing the displacements of the atmospheric models at each pixel. The merit function is evaluated at the $200 \mathrm{~km}$ height level. At this layer the response function of Stokes $\mathrm{V}$ of the visible lines to the magnetic field strength reaches its maximum and consequently the uncertainties of the inverted magnetic field are minimal. Besides, at this layer the error introduced by neglecting the acceleration term in equation (5) is expected to be smaller than at deeper photospheric layers since, in sunspot penumbrae, the velocity field is confined to lower layers.

Owing the uncertainties of the inversion method, the obtained stratification of the magnetic field is neither solenoidal nor does it satisfy the equation of motion. Thus, for the evaluation of $\chi^{2}$ we use slightly modified values for the magnetic field vector inside its uncertainties, i.e. we define $B^{\prime}(x, y, z)=B(x, y, z)+N_{B}(x, y)$, $\gamma^{\prime}(x, y, z)=\gamma(x, y, z)+N_{\gamma}(x, y), \quad$ and $\quad \phi^{\prime}(x, y, z)=$ 
$\phi(x, y, z)+N_{\phi}(x, y)$, with absolute values of noise functions $N_{B}(x, y), \quad N_{\gamma}(x, y), \quad N_{\phi}(x, y)$ smaller than the error uncertainties of the respective parameters. The resulting synthesized Stokes profiles, considering $B^{\prime}, \gamma^{\prime}$ and $\phi^{\prime}$, are still compatible with the observed Stokes profiles. In summary, the solution found by the genetic algorithm is constituted by the optimum values of $D_{z}(x, y), N_{B}(x, y), N_{\gamma}(x, y)$, and $N_{\phi}(x, y)$. We have performed several realizations of the genetic algorithm to determine the optimum values of the weights $w_{1}$, $w_{2}$. If we set $w_{1}$ to zero (i.e. just minimizing $\nabla \cdot \vec{B}$ ) many different solutions are reached; with $w_{2}$ equal to zero (i.e., minimizing the components of $\vec{F}$ ) the solution reduces in particular the $\nabla \cdot \vec{B}$ values, however not satisfactorily. The best solution is reached setting $w_{1}$ and $w_{2}$ such that both addends in equation (8) contribute in an equal manner $\left(w_{2}=3.3 \cdot 10^{5} \cdot w_{1}\right.$, using cgs units $)$. In each realization, the code produces slightly different results with an approximately Gaussian distribution around a mean value at each pixel. Therefore we adopted the average of 20 individual realizations as the final solution. In the upper left panel of Figure 5 we present the resulting displacement $D_{\mathrm{z}}(x, y)$. The displacements span a range between $-250 \mathrm{~km}$ and $100 \mathrm{~km}$ and show a spatial distribution well correlated with the $x-y$ maps of several physical quantities, e.g. $T$, $B$, or $\gamma$ (see Figure 3). Negative numbers in this scale correspond to deeper layers. The zero height of the scale has been arbitrarily set at one of the pixels analyzed; its comparison with an absolute height scale in the quiet Sun photosphere is discussed in Sect. 5. The relative noise $N_{B}(x, y), N_{\gamma}(x, y)$, and $N_{\phi}(x, y)$ is presented in the upper right and lower panels of Figure 5. Note that the introduced noise functions account for errors below $3 \%$ in $B$ and $\gamma$, and below $4^{\circ}$ in case of $\phi$.

After introducing $D_{\mathrm{z}}(x, y), N_{B}(x, y), N_{\gamma}(x, y)$ and $N_{\phi}(x, y)$ and interpolating to a common $z$-scale we can assume that the layer at $200 \mathrm{~km}$ approximately satisfies both, $\nabla \cdot \vec{B}$ and the equation of motion, although the pressure stratification for each pixel continues being the $\mathrm{HE}$ one. We can obtain now a more accurate $P_{\mathrm{g}}$-stratification by the integration of the $z$-component of equation (7). However, when the $P_{\mathrm{g}}$-stratification changes, the $z$-scale is also modified. Provided that $T(\tau)$ and $B(\tau)$ are invariant, it is easier to integrate this equation in terms of optical depth. We can rewrite the vertical component of equation (7) in terms of $\tau$, setting $\vec{F}=0$ :

$$
\frac{d P_{g}}{d \tau}=\frac{g}{\kappa}-\frac{R T}{\kappa \mu P_{g}}(\vec{J} \times \vec{B})_{\mathrm{z}} .
$$

After the integration of this equation, e.g. by RungeKutta, we obtain $P_{\mathrm{g}}$. From the equation of state we evaluate $\rho$ and subsequently the new $z$-scale from equation (11). The newly obtained $P_{g}$ values produce slightly modified stratification and the whole procedure starting with equation (7) is again repeated. The modifications are minimal and convergence is achieved after two iterations. Figure 6] shows the histograms of the divergence of the magnetic field and of the three components of the residual force before and after the minimization. The process clearly improves $\nabla \cdot \vec{B}$ (changing the standard deviation of its histogram, $\sigma$, from 0.38 to $0.16 \mathrm{G} / \mathrm{km}$ ),
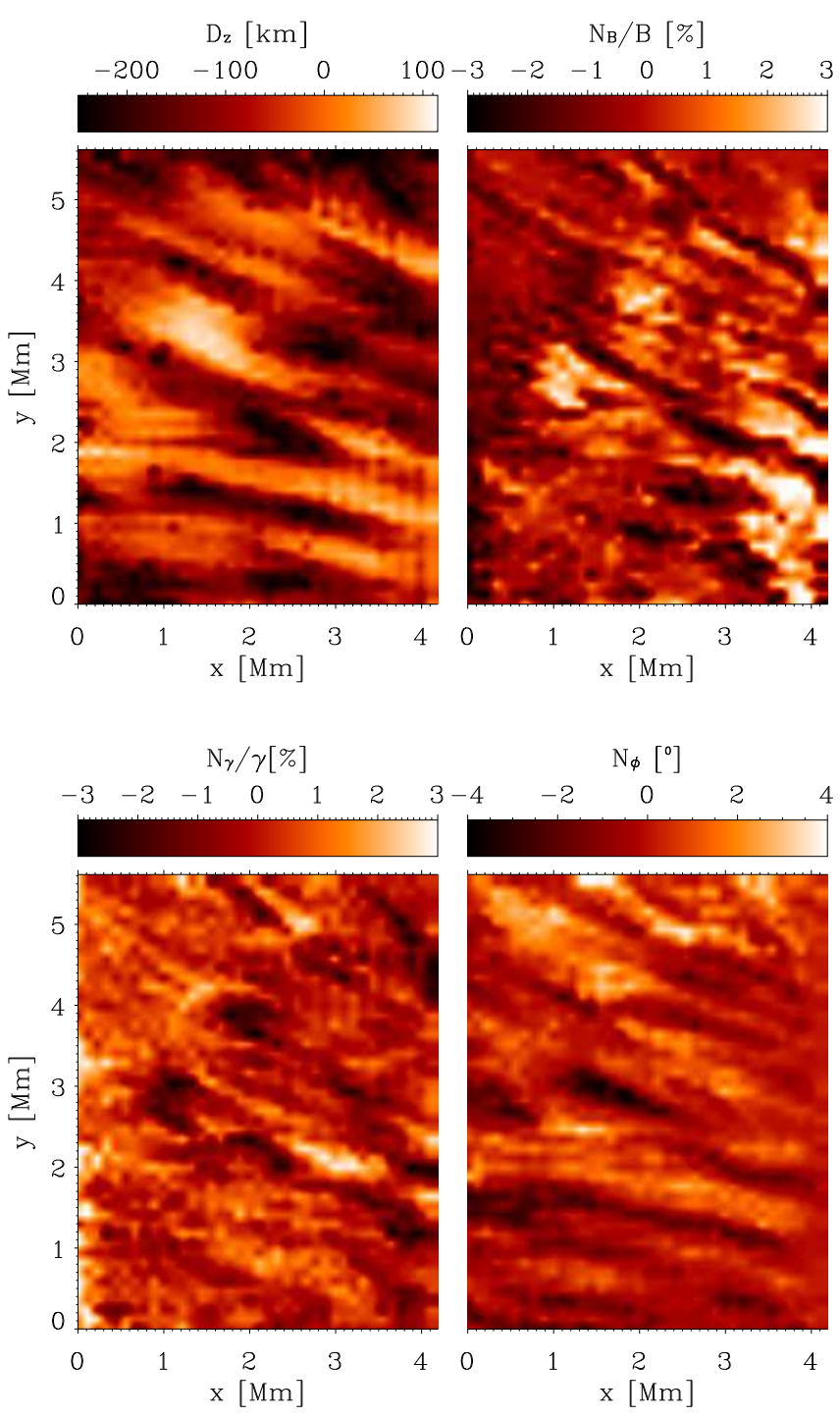

FIG. 5.- Solutions of the genetic algorithm: Displacement of the $z=200 \mathrm{~km}$ layer $D_{\mathrm{z}}(x, y)$ and noise functions $N_{B}, N_{\gamma}$, and $N_{\phi}$. See text for details. We do not plot relative $N_{\phi}$ values because $\phi$ reaches null values at some pixels.

$F_{\mathrm{x}}\left(\sigma\right.$ from $8 \cdot 10^{-4}$ to $\left.4 \cdot 10^{-4} \mathrm{dyn} / \mathrm{cm}^{3}\right)$, and $F_{\mathrm{y}}(\sigma$ from $8 \cdot 10^{-4}$ to $\left.6 \cdot 10^{-4} \mathrm{dyn} / \mathrm{cm}^{3}\right)$ and slightly diminishes $F_{\mathrm{z}}(\sigma$ from $6 \cdot 10^{-4}$ to $5 \cdot 10^{-4} \mathrm{dyn} / \mathrm{cm}^{3}$ ).

The models at all pixels were then interpolated to a common global $z$-scale. In Figure 7 we present the resulting histograms of $\nabla \cdot \vec{B}$ at six different layers. To check the significance of the results, we evaluated histograms of the simulated magnetic field distributions which deviate from solenoidal fields only by Gaussian noise, with a sigma equal to the estimated errors of the field components at each layer. We plot the mean histograms of 1000 realizations shifted by $0.2 \mathrm{G} / \mathrm{km}$ as red lines. Owing to the resulting coincidence between the histograms of both $\nabla \cdot \vec{B}$ and the errors, we conclude that in the range $50 \mathrm{~km}$ to $200 \mathrm{~km}$, the residual value of $\nabla \cdot \vec{B}$ can be entirely attributed to noise except for a systematic offset of $0.2 \mathrm{G} / \mathrm{km}$. At lower and higher layers, 

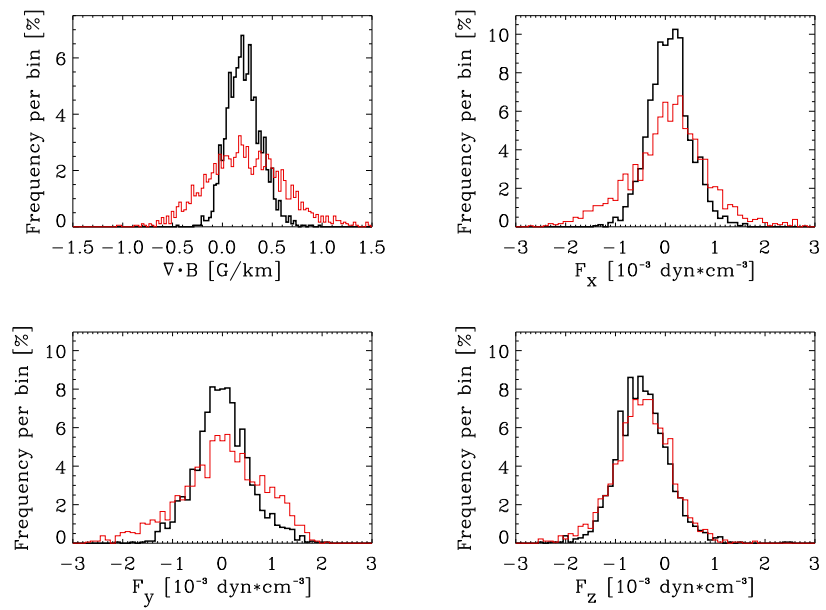

FIG. 6.- Histograms of $\nabla \cdot \vec{B}$ and the three components of the residual force $\vec{F}$ at $z=200 \mathrm{~km}$ before (red lines) and after (black lines) the application of the genetic algorithm. Binsize of 0.025 $(\nabla \cdot \vec{B})$ and $10^{-4}$ (force components), respectively.
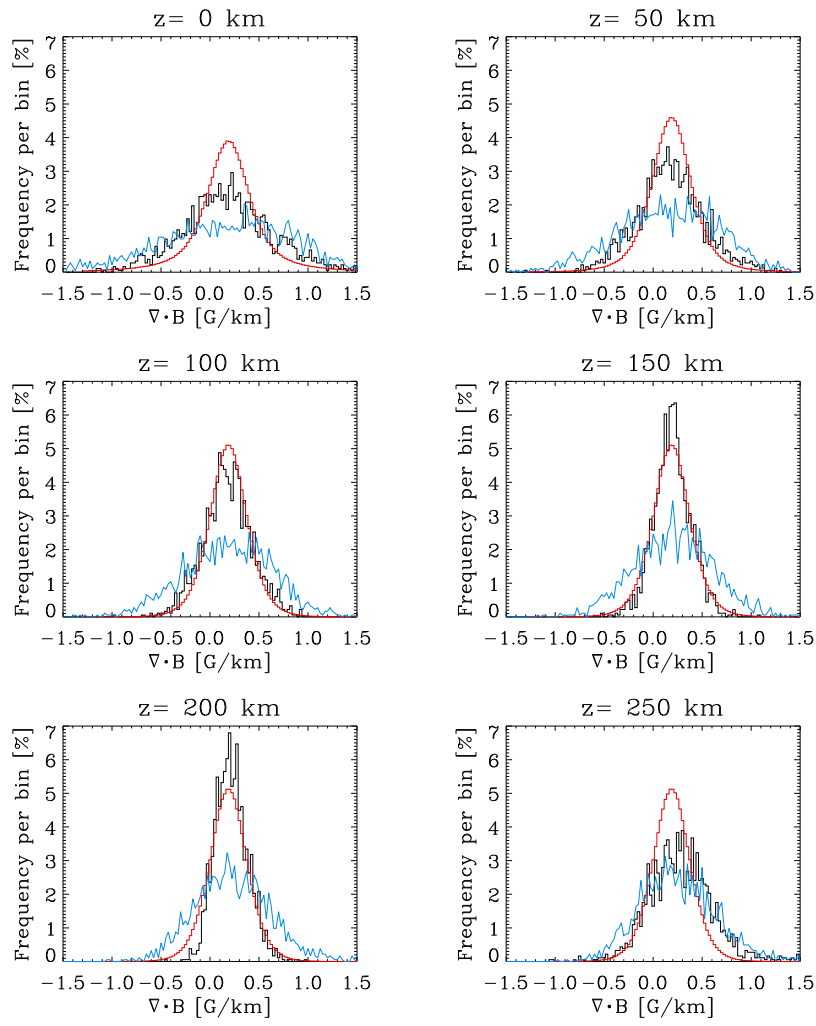

FIG. 7.- Black: Histograms of the divergence of the magnetic field strength at six different layers after the integration of the vertical component of the equation of motion. Red: Mean histograms of the divergence obtained from a random Gaussian field distribution. The error histograms have been shifted by $0.2 \mathrm{G} / \mathrm{km}$. Blue: Histograms obtained before the application of the genetic algorithm. Binsize of 0.025

not all $\nabla \cdot \vec{B}$ values can be regarded as noise. The blue lines represent $\nabla \cdot \vec{B}$ calculated with a $z$-scale obtained in hydrostatic equilibrium and considering as boundary condition $Z_{\mathrm{W}}=0$ and $P_{g}(\log (\tau)=-4.8)=655 \mathrm{dyn}^{-2}$. Note that at layers below $250 \mathrm{~km}$ the divergence of the magnetic field vector improves considerably after applying the genetic algorithm.

What remains elusive is the origin of the systematic $0.2 \mathrm{G} / \mathrm{km}$ offset. To understand how this value is distributed among the three components of $\nabla \cdot \vec{B}$, the horizontal derivatives $\frac{\partial B_{\mathrm{x}}}{\partial x}, \frac{\partial B_{\mathrm{y}}}{\partial y}$ and the vertical derivative $\frac{\partial B_{z}}{\partial z}$, in Figure 8 we show these components separately (both before and after the minimization of the merit function by the genetic algorithm). It turns out to be somewhat surprising that the individual histograms of the derivatives in the three Cartesian directions do not change much with the application of the minimization algorithm. It is only their addition in the form of the divergence that is effectively minimized by the algorithm. But in this figure, we readily see that the offset of $0.2 \mathrm{G} / \mathrm{km}$ originates entirely from the vertical derivative. This should be no surprise. Already Westendorp Plaza et al. (2001a), who performed a similar analysis to the one done in this work but without computing a geometrical height scale, found an excess in the vertical derivative after inverting data obtained with the Advanced Stokes Polarimeter (see Lites et al. 1993). These data had a spatial resolution at least three times worse than that used in our work. Westendorp Plaza et al. (2001a) attributed the origin of this excess to the unresolved nature of the structuring of the penumbral magnetic field. Interestingly, they reported on a mean excess 5-10 times larger than the $0.2 \mathrm{G} / \mathrm{km}$ found here. The question of how the vertical derivative can be made compatible with the null divergence condition was debated by several authors. Sánchez Almeida (1998) suggested that a microstructuring of the penumbral magnetic field in ranges around $1-10 \mathrm{~km}$ is able to solve this problem. Following Martínez Pillet (2000), the azimuthal components generated by the background field wrapping around penumbral flux tubes cancel out in the resolution element leading to the small horizontal gradients observed (see also Borrero et al. 2008). In Figure 1 of Sánchez Almeida (1998) one finds the magnitude of the horizontal derivatives that enter the calculation of the divergence for observations with resolution worse than $0^{\prime \prime} 2$. The values cited fluctuate with typical magnitudes of $0.15 \mathrm{G} / \mathrm{km}$, clearly smaller than the vertical gradients estimated with 1 arcsec resolution spectropolarimetry. The situation in our present analysis has improved considerably as now the horizontal derivatives fluctuate around values that are of the same order of magnitude than the vertical one (see Figure 8). However, the excess of $0.2 \mathrm{G} / \mathrm{km}$ still shows the presence of unresolved components that could be present either in the vertical or in the horizontal directions.

In order to estimate the compatibility of the resulting models with the observed Stokes profiles $\left(\mathrm{I}^{o b s}, \mathrm{Q}^{o b s}, \mathrm{U}^{o b s}, \mathrm{~V}^{o b s}\right)$, we have synthesized the Stokes profiles $\left(\mathrm{I}^{\text {final }}, \mathrm{Q}^{\text {final }}, \mathrm{U}^{\text {final }}, \mathrm{V}^{\text {final }}\right)$ from the final model atmospheres. In the upper left panel of Figure 9, we present the histogram of the rms difference $\Delta \mathrm{I}_{\mathrm{rms}}=\sqrt{\sum\left(\mathrm{I}^{\text {final }}-\mathrm{I}^{\text {obs }}\right)^{2}}$ between the final and the observed profiles (red line). The histogram of rms differences between the best fit found by SIR (labelled in the figure as SIR) and the observed profiles are plotted in 

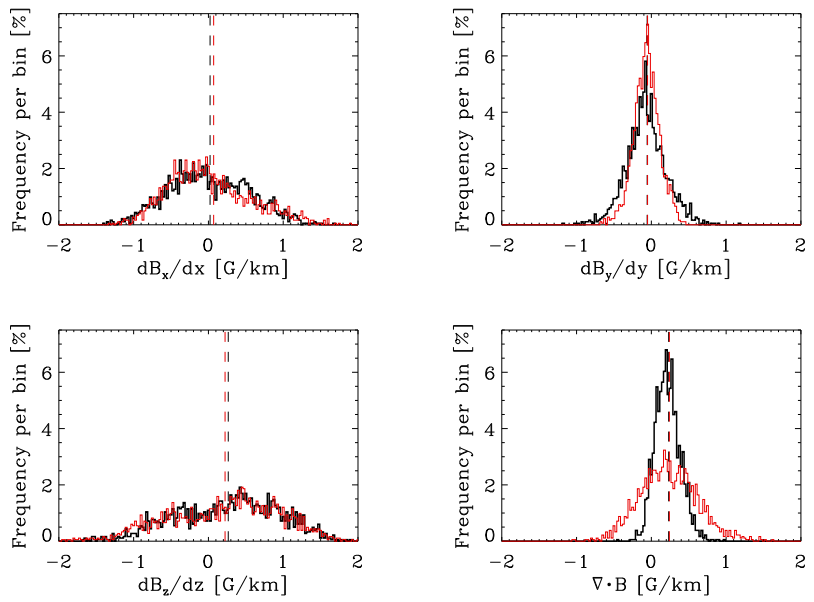

FIG. 8.- Horizontal (top) and vertical (bottom left) derivatives both before (red) and after (black) the minimization by the genetic algorithm. Vertical dashed lines correspond to the mean values of the distributions. The bottom right panel shows the sum of the three derivatives and is the same histogram as shown in Figure 6] at other scale.
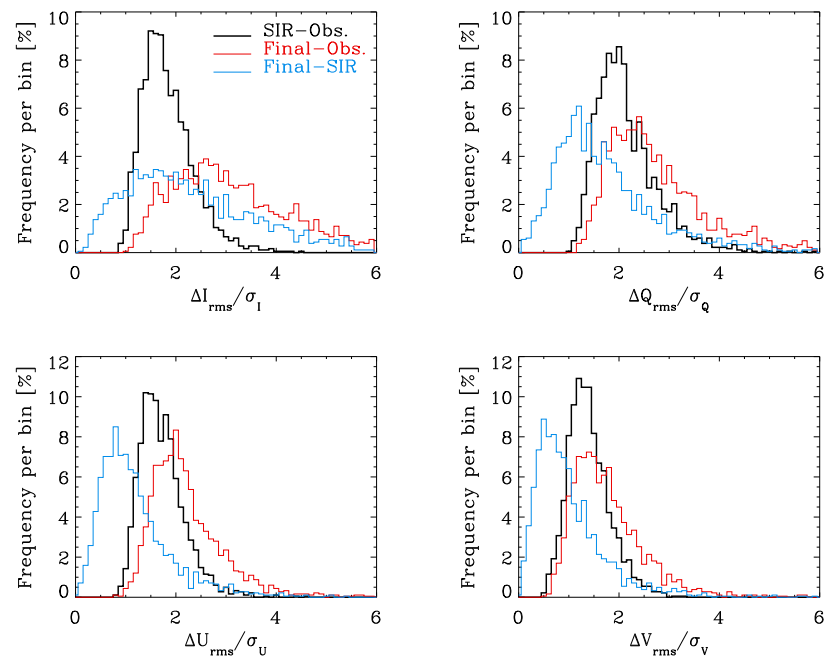

FIG. 9.- Histograms of the rms differences between the inverted (SIR) and the observed (Obs.) Stokes profiles in units of the standard deviation of the noise for the respective Stokes parameter (black). Histograms of the rms differences between the Stokes profiles synthesized from the final models (Final) and the observed profiles (red) and between the final and the SIR profiles (blue).

black. This rms is precisely the quantity minimized by the inversion method. The histogram of rms differences between the final and the SIR profiles (i.e. the error introduced by the modification of the model through the application of the present technique) is presented in blue. The corresponding histograms for the other Stokes parameters are shown in the remaining panels of this figure. Note that the rms differences between the SIR and the observed profiles peak around $1.5 \sigma-2 \sigma$ (where $\sigma$ is the standard deviation of the noise in the respective observed Stokes parameter), while the additional noise introduced by the present technique has a distribution that peaks (in case of $\mathrm{Q}, \mathrm{U}$, and $\mathrm{V}$ ) at around $1 \sigma$. This is com- patible with the fact that the noise functions, $N_{B}(x, y)$, $N_{\gamma}(x, y), N_{\phi}(x, y)$ introduced, are within the error uncertainties of the respective parameters. In case of Stokes I, the introduced error is slightly larger, peaking around $2 \sigma$. This enhanced sensitivity of I is due to the change of continuum intensity introduced by gas pressure modifications.

\section{RESULTS AND DISCUSSION}

In Figure 10, we present horizontal slices of different physical quantities at three different height layers, i.e. $T, B, \gamma, v_{\mathrm{z}}$, at $0 \mathrm{~km}, 100 \mathrm{~km}$, and $200 \mathrm{~km}$, respectively (the most right panels of each row will be described later). White contour lines correspond to $v_{\mathrm{z}}$ larger than $0.3 \mathrm{kms}^{-1}$ (i.e., relatively strong upflows) as estimated at $0 \mathrm{~km}$. In all panels of this figure, the typical filamentary structure of the penumbra is clearly observed, showing a qualitatively good correlation between the different physical quantities. The temperature contrast strongly diminishes with height, being nearly isothermal at $z=200 \mathrm{~km} ; \sigma(T) / \bar{T}$ changes from $11 \%$ at $z=0 \mathrm{~km}$ to $3.7 \%$ at $z=200 \mathrm{~km}$. The mean magnetic field strength slightly diminishes from $1859 \mathrm{G}$ to $1794 \mathrm{G}$ in the same height range, while the mean field inclination increases by $3^{\circ} \cdot \bar{v}_{\mathrm{z}}$ changes from an upflow of $0.22 \mathrm{~km} \mathrm{~s}^{-1}$ to zero. Areas with ascending velocities larger than $0.3 \mathrm{~km} \mathrm{~s}^{-1}$ correspond to places with higher temperatures and weaker and more horizontal magnetic fields.

In Table 1, we summarize the mean value and standard deviation of some quantities grouped in terms of vertical velocity at $z=0$. If we interpret the ascending velocities as the line-of-sight component of the Evershed flow, the behavior of $T, V_{\mathrm{z}}, B$ and $\gamma$ confirms the main properties of the uncombed scenario: ascending hotter material shows weaker and more horizontal magnetic fields. Interestingly, the upflowing material shows enhanced gas pressure and densities by around $86 \%$ and $71 \%$ respectively at all layers. Our results do not show any field-free region but rather indicate only a weak reduction of the magnetic field strength (from the nearly vertical background field of $\sim 1900 \mathrm{G}$ to the more horizontal field of bright penumbral filaments of $\sim 1750 \mathrm{G}$ ) in the observed penumbral region. The gradient of gas pressure, appearing at the borders of ascending areas, can not be compensated by the small variation of the magnetic pressure generated by the magnetic field weakening: it is balanced by the magnetic tension, which is produced by the strong variation of the magnetic field inclination. This is the reason why the method to establish an absolute geometrical height based on the equal total pressure between adjacent pixels (Sánchez Almeida 2005) can produce erroneous solutions. In Figure 11 we show a map of the gas pressure at $z=0$; the contour lines, enclosing areas harboring strong upflows, are clearly related to areas with increased gas pressure. The horizontal component of the Lorentz force (blue arrows) nearly balances the gas pressure gradient.

The weak reduction of the magnetic field strength is in contradiction with the gappy model (Scharmer \& Spruit 2006 and Spruit \& Scharmer 2006) that predicts "nearly" field-free gaps harboring convective motions and reaching photospheric layers. A possible explanation could be a lack of spatial resolution: Scharmer et al. (2008) 
TABLE 1

Mean value and standard deviation of temperature $T$, magnetic field Strength $B$, Field inclination $\gamma$, gas pressure $P_{\mathrm{g}}$, DENSITY $\rho$, AT THREE DIFFERENT HEIGHT LAYERS $(0 \mathrm{KM}, 100 \mathrm{KM}, 200 \mathrm{KM})$ FOR POINTS HARBORING VERTICAL VELOCITIES AT $z=0 \mathrm{KM}\left(v_{0}\right)$ SMALLER OR LARGER THAN $0.3 \mathrm{KM} \mathrm{S}^{-1}$, RESPECTIVELY.

\begin{tabular}{ccccccc}
\hline & Weak up/downflows & & & & & \\
$z[\mathrm{~km}]$ & Strong upflows & $T[\mathrm{~K}]$ & $B[\mathrm{G}]$ & $\gamma\left[{ }^{\circ}\right]$ & $P_{\mathrm{g}}\left[10^{3} \mathrm{dyn} \mathrm{cm}^{-2}\right]$ & $\rho\left[10^{-7} \mathrm{~g} \mathrm{~cm}^{-3}\right]$ \\
\hline \multirow{2}{*}{0} & $v_{0}<0.3 \mathrm{~km} \mathrm{~s}^{-1}$ & $5175 \pm 471$ & $1895 \pm 200$ & $119 \pm 11$ & $66 \pm 49$ & $1.9 \pm 1.2$ \\
& $v_{0}>0.3 \mathrm{~km} \mathrm{~s}^{-1}$ & $5769 \pm 614$ & $1777 \pm 177$ & $110 \pm 7$ & $125 \pm 47$ & $3.3 \pm 1.0$ \\
\hline \multirow{2}{*}{100} & $v_{0}<0.3 \mathrm{~km} \mathrm{~s}^{-1}$ & $4802 \pm 203$ & $1851 \pm 231$ & $120 \pm 9$ & $30 \pm 23$ & $1.0 \pm 0.7$ \\
& $v_{0}>0.3 \mathrm{~km} \mathrm{~s}^{-1}$ & $5001 \pm 204$ & $1763 \pm 166$ & $112 \pm 6$ & $57 \pm 24$ & $1.8 \pm 0.7$ \\
\hline \multirow{2}{*}{200} & $v_{0}<0.3 \mathrm{~km} \mathrm{~s}^{-1}$ & $4557 \pm 172$ & $1810 \pm 207$ & $121 \pm 9$ & $14 \pm 10$ & $0.5 \pm 0.3$ \\
& $v_{0}>0.3 \mathrm{~km} \mathrm{~s}^{-1}$ & $4695 \pm 123$ & $1755 \pm 163$ & $115 \pm 6$ & $25 \pm 11$ & $0.8 \pm 0.3$ \\
\hline
\end{tabular}

estimate that the minimum magnetic field strength in a dark cored penumbral filament is reduced by $25 \%$ when the spatial resolution is increased from 0". 3 to $0{ }^{\prime \prime} 15$. Following this order of magnitude estimation, the strength of our more horizontal fields would reduce to $\sim 1300 \mathrm{G}$, i.e. a figure still in the $\mathrm{kG}$ range like also found by Borrero \& Solanki (2010). The simulations of Rempel et al. (2009a,b) present reduced field strengths down to $700-1000 \mathrm{G}$.

Besides, we do not find any signature of overturning convection in the penumbral area observed (at our resolution). In Table 2, we present the net mass flow evaluated at three different geometrical heights: $0 \mathrm{~km}, 100 \mathrm{~km}$, $200 \mathrm{~km}$. The uncertainties have been calculated after the introduction of an artificial velocity offset of $\pm 100 \mathrm{~m} \mathrm{~s}^{-1}$ considering possible uncertainties in the wavelength calibration. The average mass flow is strongly ascending and cannot be considered as the residual of the cancellation between ascending and descending parcels: At $0 \mathrm{~km}$ and $100 \mathrm{~km}$, the upward directed mass flow is $\sim 5$ times larger than the downward one and $\sim 2$ times larger at $200 \mathrm{~km}$. Our results appear to be compatible with mass motions dominated by the Evershed flow which are mainly upwards directed in the inner penumbrae (Rimmele \& Marino 2006). In the panels of the $4^{\text {th }}$ column of Figure 10, the weak downflows almost exclusively appear in areas harboring stronger and more vertical (background) magnetic field. According to the gappy scenario, we should find downflows only at the borders of, but still inside, features with reduced and more horizontal magnetic fields. Perhaps, the lack of these downflows might be explained again by insufficient spatial resolution: Zakharov et al. (2008), analyzing data obtained at the 1-m Swedish Solar telescope, found weak downflows of around $90 \mathrm{~m} / \mathrm{s}$ (certainly at the limit of the velocity accuracy) at the side of one filament. Although, it is questionable wether these weak downflows can be significant enough to counterbalance the factor 5 between $\left\langle\rho v_{\mathrm{z}}\right\rangle_{\text {up }}$ and $\left\langle\rho v_{\mathrm{z}}\right\rangle_{\text {down }}$ found in the present work.

The uncombed penumbral model and the gappy model differ mainly in the way the energy is transferred. In the uncombed model the energy is carried by the Evershed flow (Ruiz Cobo \& Bellot Rubio 2008), while the gappy model roots on convective energy transfer in field free regions (Scharmer \& Spruit 2006). For the first time we are able to estimate the energy flux stratification directly from observational data. The stratification of physical quantities in common geometrical height scale delivers
TABLE 2

MeAN VAlue of Net MASS FlOW OVER THE Full OBSERVED AREA $\left.\left(<\rho v_{\mathrm{z}}\right\rangle\right)$, MEAN VALUE OF MASS FLOW IN ASCENDING $\left.\left(<\rho v_{\mathrm{z}}\right\rangle_{\text {up }}\right)$ AND DESCENDING $\left(\left\langle\rho v_{\mathrm{z}}\right\rangle_{\text {down }}\right)$ AREAS. MASS FLOW GIVEN IN units of $\left[\mathrm{g} \mathrm{s}^{-1} \mathrm{~m}^{-2}\right]$. Percentage of ascending areas (N[\%]).

\begin{tabular}{rcccc}
\hline$z[\mathrm{~km}]$ & $\left\langle\rho v_{\mathrm{z}}\right\rangle$ & $\left\langle\rho v_{\mathrm{z}}\right\rangle_{\mathrm{up}}$ & $\left\langle\rho v_{\mathrm{z}}\right\rangle_{\text {down }}$ & $\mathrm{N}[\%]$ \\
\hline 0 & $72 \pm 23$ & $96 \pm 8$ & $-17 \pm 4$ & $78 \pm 22$ \\
\hline 100 & $20 \pm 12$ & $32 \pm 2$ & $-6 \pm 4$ & $70 \pm 25$ \\
\hline 200 & $2 \pm 5$ & $9 \pm 2$ & $-5 \pm 1$ & $52 \pm 28$ \\
\hline
\end{tabular}

all the ingredients needed for this calculation. In the right panels of Figure 10 we show the energy flux (power per surface unity) $F_{\mathrm{E}}$ carried by the ascending material between two height layers in units of the solar flux $F_{\odot}=\sigma T_{\text {eff }}^{4}$. We have evaluated $F_{\mathrm{E}}$ by the integration of

$$
\frac{\partial F_{\mathrm{E}}}{\partial z}=-\rho c_{\mathrm{p}} v_{\mathrm{z}}\left[\frac{d T}{d z}-\left(\frac{d T}{d z}\right)_{a}\right]
$$

where $c_{\mathrm{p}}$ is the specific heat at constant pressure and the adiabatic temperature gradient is evaluated as

$$
\left(\frac{d T}{d z}\right)_{a}=T \frac{d \log P_{\mathrm{g}}}{d z} \nabla_{a},
$$

with $\nabla_{a}$ is the double logarithmic isentropic temperature gradient. Both $c_{\mathrm{p}}$ and $\nabla_{a}$ have been evaluated taking into account partial ionization of hydrogen.

In the upper right panel of Figure 10, $F_{\mathrm{E}}$ integrated from $z=-75 \mathrm{~km}$ to $z=0 \mathrm{~km}$ is depicted in units of solar flux. Only in areas with strong upflows (white contours), does the energy flux reach significant values and even exceed (in some points) the solar flux up to 5 times (the right hand panels of Figure 10 are not scaled to the respective $\min / \max$ value for contrast enhancement). A strong decrease of the energy flux with height is apparent. The contribution of the $0 \mathrm{~km}$ to $100 \mathrm{~km}$ layer to the energy flux reaches maximum values equal to the solar flux (medium right panel), while $F_{\mathrm{E}}$ integrated from $z=100 \mathrm{~km}$ and $z=200 \mathrm{~km}$, (bottom right panel) changes its sign, indicating that the ascending plasma is increasing its energy from the environment.

Between $z=-75 \mathrm{~km}$ and $z=0 \mathrm{~km}$ the flux averaged over the area under study amounts to $23 \%$ of the solar flux, while the medium and upper layers contribute with $0.9 \%$ and $-0.2 \%$, respectively. We determine the total amount of energy flux carried by the ascending material in our 


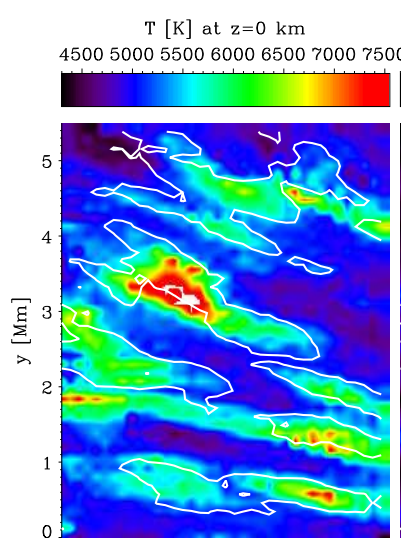

$B$ [G] at $\mathrm{z}=0 \mathrm{~km}$

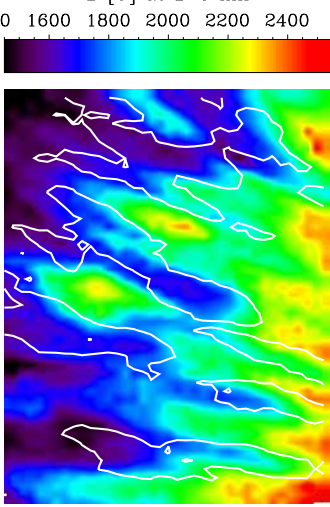

$\mathrm{T}[\mathrm{K}]$ at $\mathrm{z}=100 \mathrm{~km}$

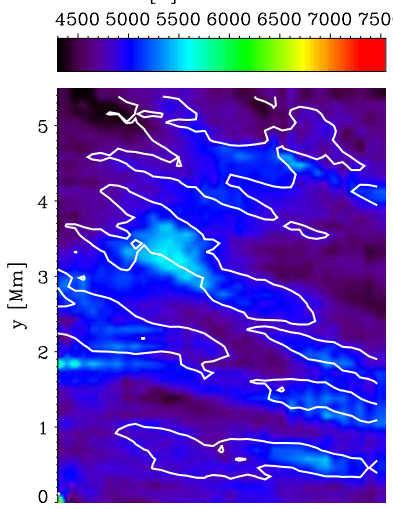

B [G] at $\mathrm{z}=100 \mathrm{~km}$
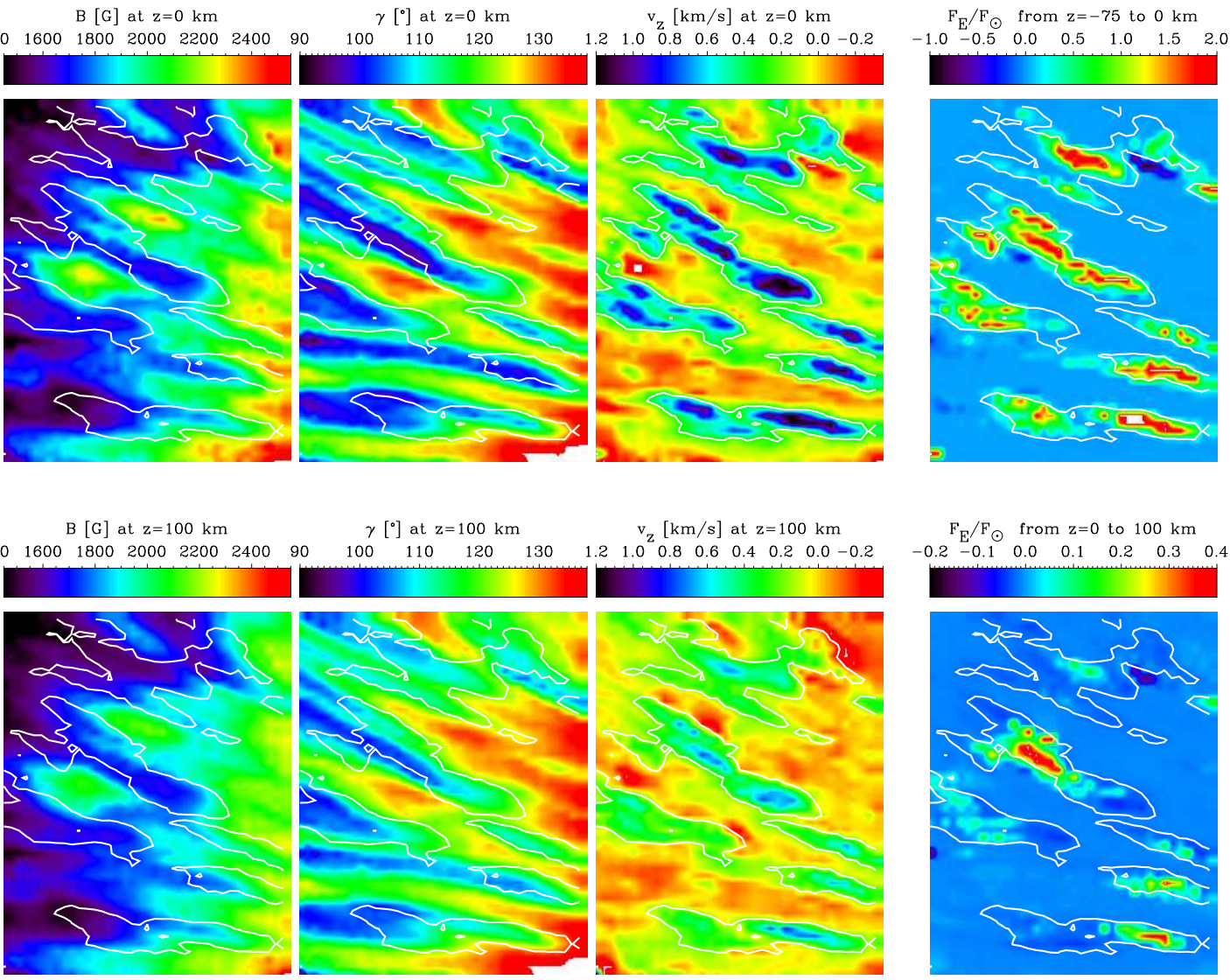

$\mathrm{F}_{\mathrm{E}} / \mathrm{F}_{\odot}$ from $\mathrm{z}=0$ to $100 \mathrm{~km}$
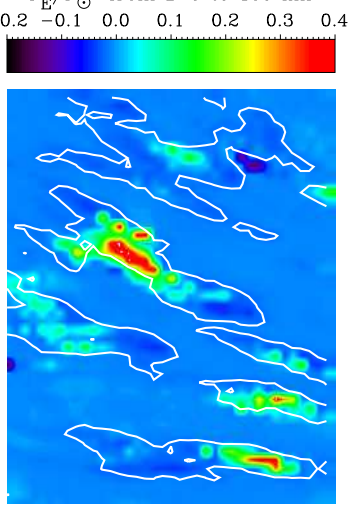

$\mathrm{T}[\mathrm{K}]$ at $\mathrm{z}=200 \mathrm{~km}$

B [G] at $\mathrm{z}=200 \mathrm{~km}$

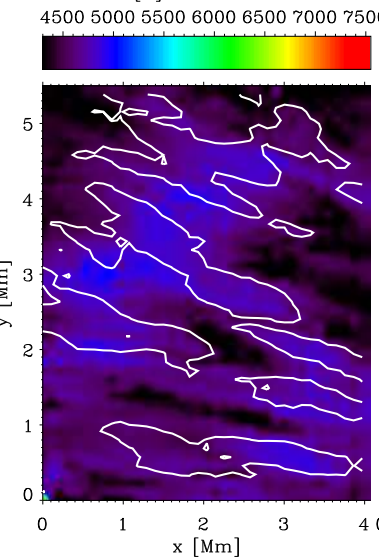

$\gamma\left[^{\circ}\right]$ at $\mathrm{z}=200 \mathrm{~km}$

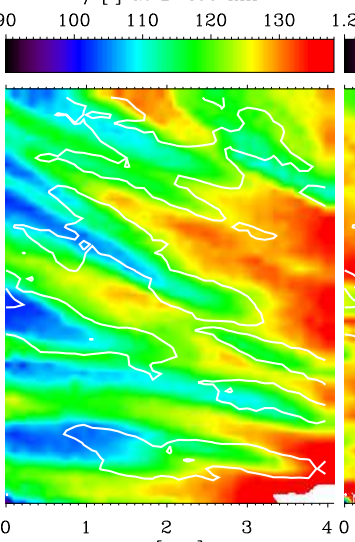

$\mathrm{v}_{\mathrm{z}}[\mathrm{km} / \mathrm{s}]$ at $\mathrm{z}=200 \mathrm{~km}$
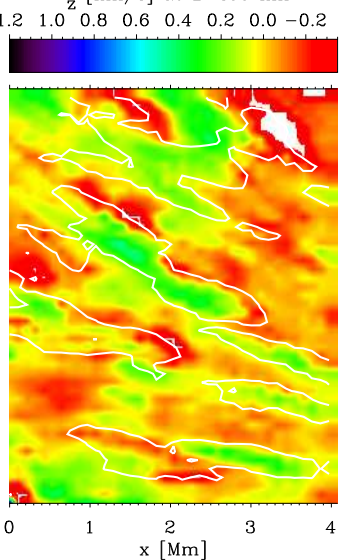

$\mathrm{F} / \mathrm{F}$ from $\mathrm{z}=100$ to $200 \mathrm{~km}$

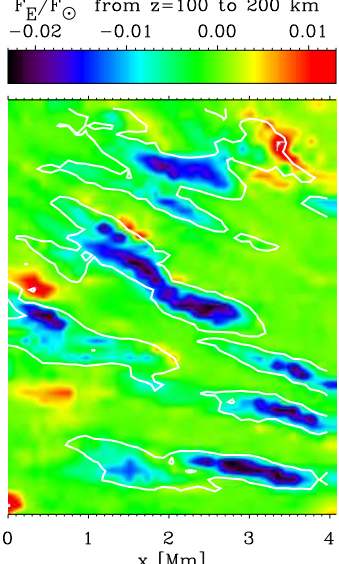

FIG. 10. - Maps of temperature $T$, magnetic field strength $B$, field inclination $\gamma$, and vertical component of velocity $v_{\mathrm{z}}$ at three different geometrical heights: $z=0 \mathrm{~km}$ (upper panels), $z=100 \mathrm{~km}$ (middle panels), $z=200 \mathrm{~km}$ (lower panels). The power per surface unity, $F_{\mathrm{E}}$, transferred by plasma motions in vertical direction between $z=-75 \mathrm{~km}$ and $z=0 \mathrm{~km}$ (upper right panel), between $z=0 \mathrm{~km}$ and $z=100 \mathrm{~km}$ (middle right panel), and between $z=100 \mathrm{~km}$ and $z=200 \mathrm{~km}$ (bottom right panel) is presented in units of the solar flux, respectively. White iso-contours enclose areas with velocities at the $z=0 \mathrm{~km}$ level larger than $0.3 \mathrm{~km} \mathrm{~s}^{-1}$ (strong upflows).

full height range to be $24 \%$ which is well below the expected $75 \%$ in sunspot penumbrae. Owing to the strong increase of the energy flux with depth one can expect that the contribution of layers below $z=-75 \mathrm{~km}$ might be sufficient to explain the observed brightness of penumbra as channeled by the upflows.

As an order of magnitude estimate we have evaluated the contribution to the energy flux $F_{\mathrm{E}}$ of the deep layers located between $z=-225 \mathrm{~km}$ and $z=-75 \mathrm{~km}$ (for reasons that will become evident later). Clearly, the visible lines used in this work are nearly insensible to physical parameters at layers deeper than $-75 \mathrm{~km}$, i.e., the stratification of temperature, density, pressure and velocity at such layers result from linear extrapolations (in the original $\log (\tau)$ scale); and so the results at these layers can be considered only qualitatively. In panel (a) of Figure 12 we plot the energy flux carried by the vertical motion of the solar plasma between $-225 \mathrm{~km}$ and $200 \mathrm{~km}$, averaged over the penumbral region studied here. The total contribution of the energy flux in the full height range 


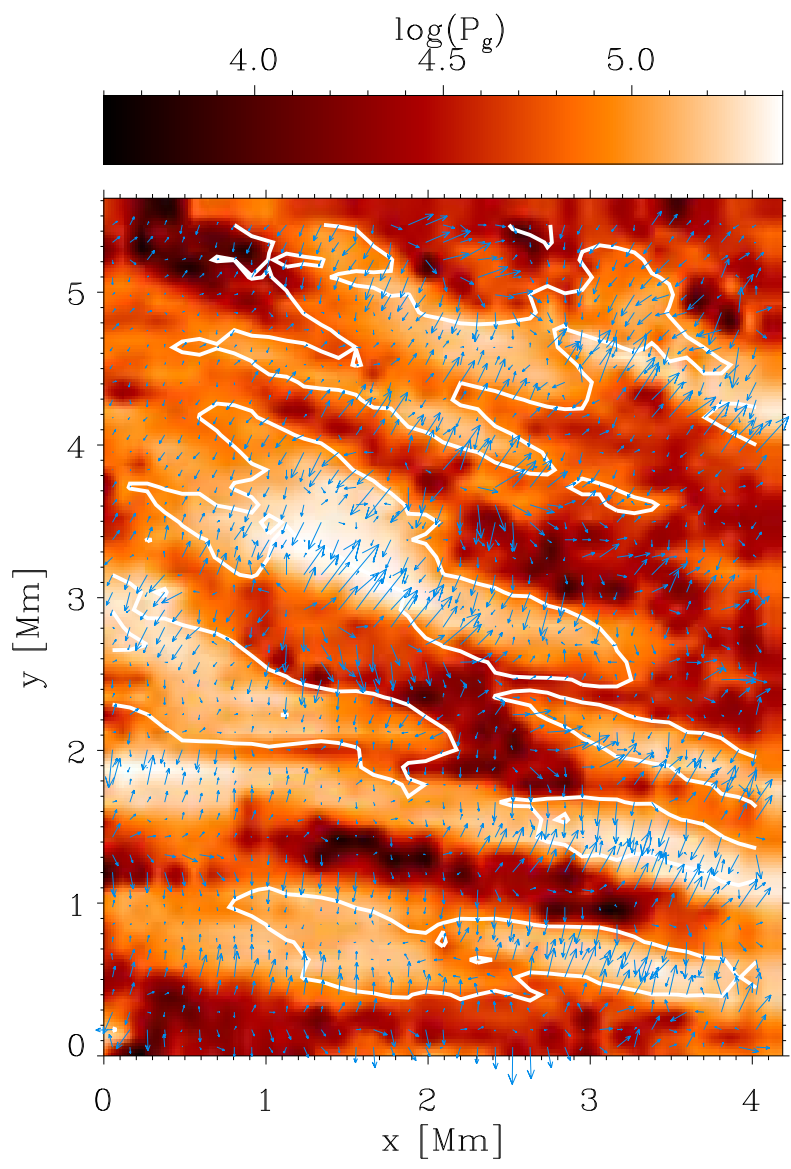

FIG. 11.- Map of gas pressure [dyn $\left.\mathrm{cm}^{-2}\right]$ and horizontal component of the Lorentz force (blue arrows) at $z=0 \mathrm{~km}$. White isocontours are the same as in Figure 10

amounts to $78.7 \%$ of the solar flux, a value near the average brightness of a sunspot penumbra. The contribution to the energy flux of layers probed by the visible lines is also plotted (in blue). At these layers, the error bars have been calculated by a Monte Carlo simulation from the uncertainties of the physical quantities produced by the inversion method and the genetic algorithm. In order to understand why only layers between $-180 \mathrm{~km}$ and $20 \mathrm{~km}$ appreciatively contribute to the energy flux, we plot in panel (b) of Figure 12 the mean temperature gradient (black) and the mean adiabatic temperature gradient (red) averaged over the region under study. The strong increase of the uncertainties of these gradients with depth reflects the above mentioned insensitivity of the lines under study at deeper layers. Nevertheless, below $0 \mathrm{~km}$ the temperature gradient is seen to be steeper than the adiabatic one, thus at these layers the ascending matter transfers energy to the surroundings. Apart from temperature gradients, it is necessary to take into account density, vertical velocity and specific heat stratification: in panel (c) of Figure 12, we plot $\left\langle-\rho c_{\mathrm{p}} v_{\mathrm{z}} \frac{d T}{d z}\right\rangle$ (black), i.e. the height derivative of the energy flux carried by the vertical component of the plasma motion under constant pressure conditions. The red line represents the correction in the gradient of energy flux which must be subtracted to account for pressure variations $<-\rho c_{\mathrm{p}} v_{\mathrm{z}}\left(\frac{d T}{d z}\right)_{a}>$. Under the caveat mentioned above
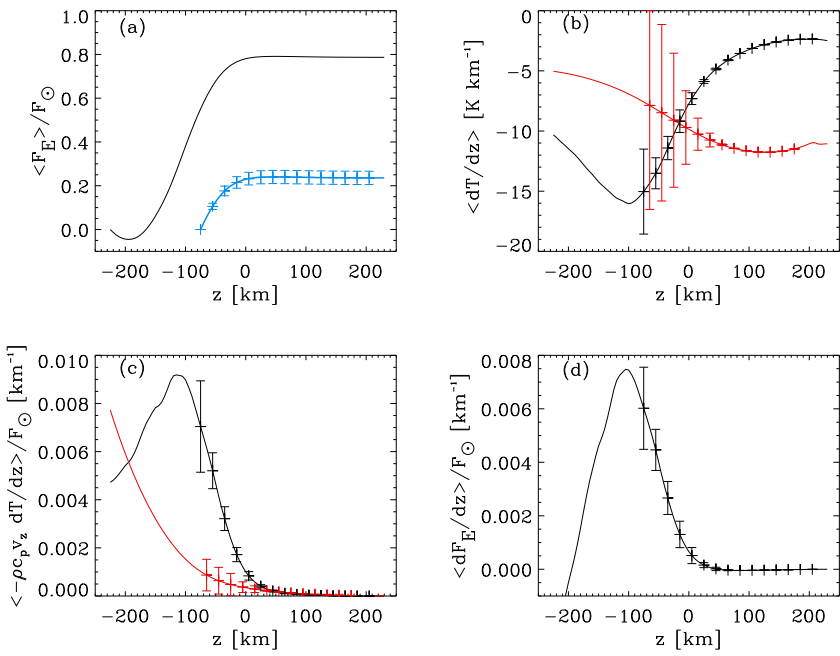

Fig. 12.- Panel (a): Energy flux $\left\langle F_{\mathrm{E}}\right\rangle$ in solar flux units evaluated from the integration of the function plotted in panel (d) from $-225 \mathrm{~km}$ until $z$. Contribution to the energy flux of layers higher than $-75 \mathrm{~km}$ together with their uncertainties (blue). Panel (b): mean temperature gradient (black) and mean adiabatic temperature gradient (red) averaged over the region under study. The error bars represent the errors of the mean temperature gradients above $-75 \mathrm{~km}$. Panel (c): Height derivative of the energy flux carried by the vertical component of the plasma motion under constant pressure conditions $\left\langle-\rho c_{\mathrm{p}} v_{\mathrm{z}} \frac{d T}{d z}>\right.$ in units of the solar flux (black), and the correction (red) in the height gradient of the energy flux which must be subtracted to account for pressure variations $<-\rho c_{\mathrm{p}} v_{\mathrm{z}}\left(\frac{d T}{d z}\right)_{a}>$. Panel (d): height derivative of the energy flux carried by the vertical component of the plasma motion in units of the solar flux.

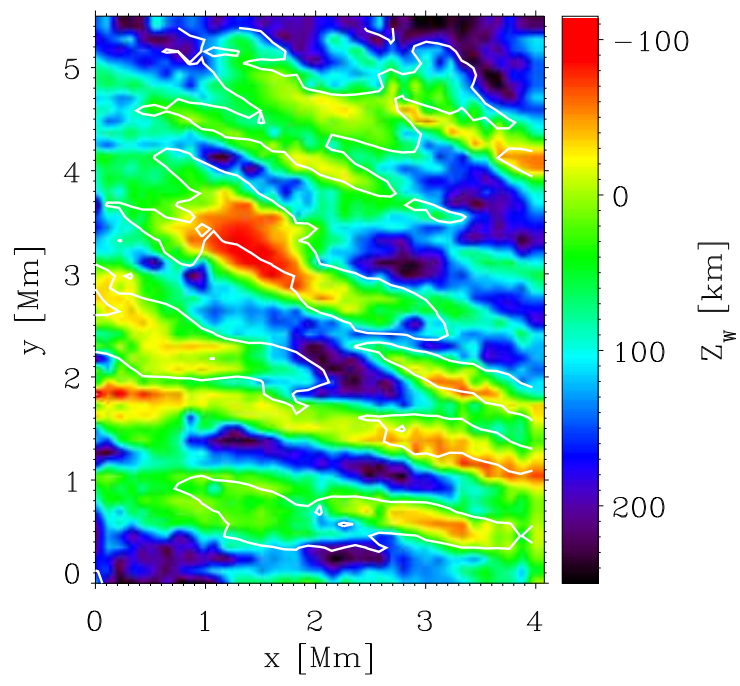

FIG. 13.- Map of Wilson depression $Z_{\mathrm{W}}$. White iso-contours enclose areas with velocities at $z=0 \mathrm{~km}$ larger than $0.3 \mathrm{~km} \mathrm{~s}^{-1}$ (strong upflows). The origin of the $Z_{\mathrm{W}}$-scale is arbitrary and $\sim 100 \mathrm{~km}$ should be added.

we can understand in the light of this figure why layers above $-200 \mathrm{~km}$ are heated by the ascending material.

In panel (d) of Figure 12, we present the height derivative of the energy flux carried by the vertical component of the plasma motion in units of the solar flux evaluated as the difference between the black and red line of panel 

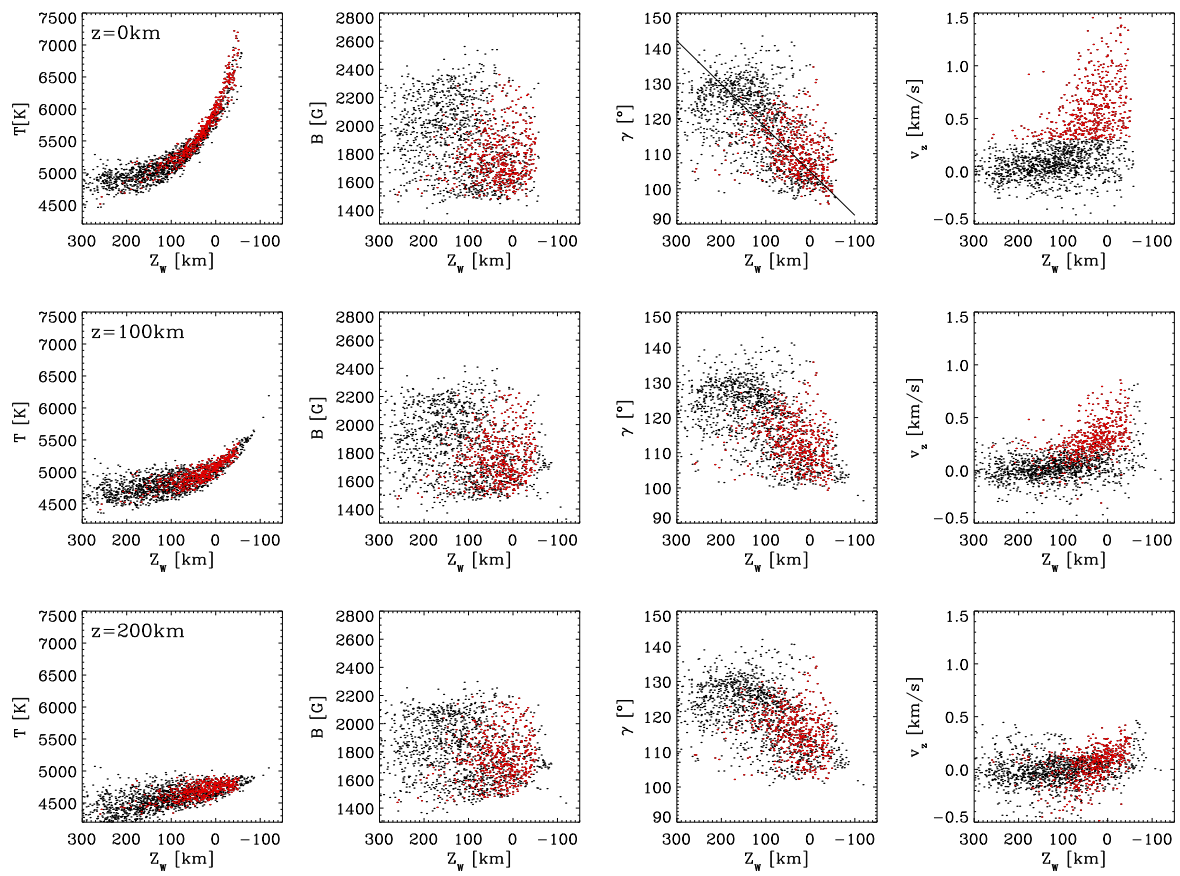

Fig. 14. - Scatter plots of temperature $T$, magnetic field strength $B$, field inclination $\gamma$, and vertical component of velocity $v_{\mathrm{z}} v s$. Wilson depression; at $z=0 \mathrm{~km}$ (upper panels), $z=100 \mathrm{~km}$ (middle panels), and $z=200 \mathrm{~km}$ (bottom panels). Points with vertical velocities at $z=0 \mathrm{~km}$ larger than $0.3 \mathrm{~km} \mathrm{~s}^{-1}$ (strong upflows) are presented in all panels by red colour. The origin of the $Z_{\mathrm{W}}$-scale is arbitrary and $\sim 100 \mathrm{~km}$ should be added.

(c), which is essentially the average of equation (10).

Another important parameter, which can be evaluated once the absolute geometrical height scale is known, is the Wilson depression, i.e. the depth where the continuum optical depth equals 1 . The knowledge of $Z_{\mathrm{W}}$ allows us to study which penumbral features are elevated or depressed. In Figure 13 we present the map of $Z_{\mathrm{W}}$. Areas harboring significant up-flows (enclosed by the iso-velocity lines) correspond to elevated structures $\left(Z_{\mathrm{W}}<100 \mathrm{~km}\right)$. When comparing with Figure 10, a good correlation with the different physical quantities is apparent. For a quantitative analysis of this correlation, scatter plots of $T, B, \gamma, v_{\text {los }}$ vs. the Wilson depression are depicted in Figure 14 for several geometrical heights. We observe a strong correlation between temperature $T$ and $Z_{\mathrm{W}}$ : in places with higher $T, \log (\tau)=0$ is displaced to upper layers due to the strong dependence of opacity with temperature (in agreement with the results found by Schmidt \& Fritz 2004). Note that, as stated above, the $z=200 \mathrm{~km}$ layer is nearly isotherm. Furthermore, for depressed areas (e.g. $Z_{\mathrm{W}}>150 \mathrm{~km}$ ) the difference in temperature between the heights of $0 \mathrm{~km}$ and $200 \mathrm{~km}$ is only $\sim 500 \mathrm{~K}$, while for elevated areas (e.g. $Z_{\mathrm{W}}<0 \mathrm{~km}$ ), temperature differences of the order of $2000 \mathrm{~K}$ between both layers are reached. The magnetic field strength $B$ shows a very weak correlation with $Z_{\mathrm{W}}$ : lower $Z_{\mathrm{W}}$ tends to be related to weaker magnetic fields. Nevertheless, we find clear trends between field inclination $\gamma$ and the Wilson depression, at all layers. The same behavior is observed in case of the vertical component of velocity $v_{\mathrm{z}}$. In Figure 10 we have plotted the points corresponding to pixels harboring significant upflows (in red). Focusing on the distribution of red points throughout the panels, we can conclude that the Evershed flow corresponds to areas with decreased Wilson depression (elevated structures), hotter temperatures, and weaker and more horizontal magnetic fields. The mean value and the standard deviation of the Wilson depression in areas with stronger ascending velocities $\left(v_{\mathrm{z}}>0.3 \mathrm{~km} \mathrm{~s}^{-1}\right)$, amount to $34 \mathrm{~km} \pm 57 \mathrm{~km}$, while in the remaining areas values of $122 \mathrm{~km} \pm 82 \mathrm{~km}$ are retrieved: i.e. features harboring the Evershed flow are in average clearly elevated.

The zero in the $z$-scale (and consequently the zero of the Wilson depression) is arbitrary. Since the area where the genetic algorithm has been applied did not include the surroundings of the spot, an absolute scale with respect to the quiet photosphere was not directly inferred from our analysis. However, such an absolute scale can be established in the following way. If we assume that the central part of the umbra has a Wilson depression of 600 $\mathrm{km}$ (Solanki 2003) with an inclination of 180 degrees, a linear continuation of the data points in the third panel of the upper row in Figure 14 (see linear fit) establishes our mean umbral $\tau=1$ level at $500 \mathrm{~km}$, suggesting that our $0 \mathrm{~km}$ reference level is $\sim 100 \mathrm{~km}$ depressed with respect to the normal quiet Sun. This indicates that our mean Wilson depression over the FOV (94 $\pm 86 \mathrm{~km}$ in our scale) corresponds to a depression of $\sim 194 \mathrm{~km}$ with respect to the surrounding quiet Sun atmosphere. This value is in agreement with previous estimates of the Wilson depression at the inner penumbra (Ruedi et al. 1995). 
Using a genetic algorithm we have established a common geometrical height scale for photospheric penumbral models resulting from SIR inversions of Hinode data. Although the genetic algorithm only minimizes $\nabla \cdot \vec{B}$ and the deviations from static equilibrium in the equation of motion at a height of $200 \mathrm{~km}$, the resulting atmospheric model, interpolated to a common $z$-scale, shows very small $\nabla \cdot \vec{B}$ values (and in any case, always inside the calculated error bars) in a height range from $50 \mathrm{~km}$ to $200 \mathrm{~km}$. The horizontal derivatives of the divergence are of similar magnitude to that of the vertical one. However, the latter has a mean value $(0.2 \mathrm{G} / \mathrm{km})$ that cannot be compensated by the mean values of the horizontal derivatives. This shows that, even at Hinode resolutions of $0.3^{\prime \prime}$ $(200 \mathrm{~km})$, the penumbra displays unresolved structure. This unresolved structure can be present in both horizontal and vertical directions. In the first case, improved spatial resolution should reduce the mean value of the vertical derivative. In the second case, the only way to achieve better resolution in the $z$-direction, would be by including more spectral lines in the inversion.

Having at our disposal a common geometrical height scale, we can evaluate e.g. electric currents (which will be analyzed in a forthcoming paper), the Wilson depression, and in general the three dimensional structure of penumbral features. The results show a significant correlation between the different physical quantities. Owing to the visible photospheric lines used, we are only able to reliably probe the penumbra in the range from $\sim 0 \mathrm{~km}$ to $\sim 200 \mathrm{~km}$. At deep layers, strong spatial contrast in all parameters is found, whereas at the highest layers both velocities and temperatures are nearly spatially homogeneous. Penumbral filaments with strong upflows have a weaker and more horizontal magnetic field, are hotter and more dense and exhibit a $\tau=1$ surface displaced to higher layers. All these properties fit into the picture proposed by the uncombed scenario.

We point out that at the resolution of the Hinode/SP there is no trace of field-free regions in the penumbral volume retrieved by observations (pixel volume of $\sim 200^{3} \mathrm{~km}^{3}$ ). Our inversions show $\mathrm{kG}$ field strength at all points inside this penumbral volume. We do not find traces of overturning convection in the inner penumbral area analyzed: the mass flow evaluated at three different height layers is clearly dominated by upflows, the amount of ascending material being five times larger than the descending one. This results might certainly change when analyzing the mid and outer penumbra due to the presence of strong downflow patches as found by Ichimoto et al. (2007a).

Besides, the observed upward motion, here identified as the vertical component of the Evershed flow, seems to carry enough energy to explain the brightness of the penumbra if the physical quantities below $z=-75 \mathrm{~km}$ are extrapolated from the results of the inversion.

As the method presented here has been applied so far only on a small penumbral region its extension on the entire sunspot penumbra, including the umbra and moat regions, will be addressed in a future work.

This work has been supported by the Spanish Ministerio de Ciencia e Innovación through projects ESP 200613030-C06-01, AYA2007-65602, AYA2007-63881, and the European Commission through the SOLAIRE Network (MTRN-CT-2006-035484). The genetic algorithm has been kindly provided by E. Páez Mañá. We thank C. Beck, R. Schlichenmaier, and S. K. Solanki for fruitfull discussion.

\section{REFERENCES}

Balthasar, H., Bello González, N., Collados, M., et al. 2009, in: Strassmeier, K.G., Kosovichev, A.G., Beckmann, J.E. (eds.), Cosmic Magnetic Fields: From Planets, to Stars and Galaxies, IAU Symp. 259, p. 665

Beck, C. 2006, Ph.D.Thesis, Albert-Ludwigs-University, Freiburg Beck, C. 2008, A\&A, 480, 825

Bello González, N. \& Kneer, F. 2008, A\&A, 480, 265

Bello González, N., Okunev, O. V., Domínguez Cerdeña, I., Kneer, F., \& Puschmann, K. G. 2005, A\&A, 434, 317

Bellot Rubio, L., in Magnetic coupling between the Interior and the Atmosphere of the Sun, S.S. Hassan and Rutten (eds.), ASP Ser., 2009, in press, arXiv:0903.3619

Bellot Rubio, L. R., Balthasar, H., \& Collados, M. 2004, A\&A, 427, 319

Borrero, J. M. 2007, A\&A, 471, 967

Borrero, J. M. 2009, Sci. China Ser. G, 52, 1670

Borrero, J. M., Lagg, A., Solanki, S. K., \& Collados, M. 2005 , A\&A, 436, 333

Borrero, J. M., Lites, B. W., \& Solanki, S. 2008, A\&A, 481, L13

Borrero, J. M. \& Solanki, S. K. 2010, ApJ, 709, 349

Borrero, J. M., Solanki, S. K., Lagg, A., Socas-Navarro, H., \& Lites, B. 2006, A\&A, 436, 333

Brummell, N. H., Tobias, S. M., Thomas, J. H., \& Weiss, N. O. 2008, ApJ, 686, 1454

Carroll, T. A. \& Kopf, M. 2008, A\&A, 481, L37

Denker, C. Deng, N. Rimmele, T. R. Tritschler, A. Verdoni, A. 2007, Sol. Phys., 241, 411D

Frutiger, C., Solanki, S. K., Fligge, M., \& Bruls, J. H. M. J. 2000 A\&A, 358, 1109

Gizon, L., Schunker, H., Baldner, C. S., et al. 2009, Space Sci. Reviews, 144, 249

Heinemann, T., Nordlund, Å., Scharmer, G. B., \& Spruit, H. C. 2007, ApJ, 669, 1390

Ichimoto, K., Shine, R. A., Lites, B., et al. 2007a, PASJ, 59, 593
Ichimoto, K., Suematsu, Y., Tsuneta, S., et al. 2007b, Science, 318,1597

Jurcák, J. \& Bellot Rubio, L. 2008, A\&A, 481, L17

Jurcák, J., Bellot Rubio, L., Ichimoto, K., et al. 2007, PASJ, 59, 601

Langhans, S., Scharmer, G. B., Kiselman, D., \& Löfdahl, M. G. 2007, A\&A, 464, 763

Lites, B. W., Elmore, D. F., Seagraves, P., \& Skumanich, A. 1993, ApJ, 418, 928

Lites, B. W., Elmore, D. F., \& Streander, K. V. 2001, ASPC, 236, $33 \mathrm{~L}$

Löfdahl, M. G. 2002, Proc. SPIE, 4792, 146

Mathew, S. K., Lagg, A., Solanki, S. K., et al. 2003, A\&A, 410, 695

Martínez Pillet, V. 2000, A\&A, 361, 734

Martínez Pillet, V., Katsukawa, Y., Puschmann, K. G., \& Ruiz Cobo, B. 2009, ApJ, 701, L79

Müller, D. A. N., Schlichenmaier, R., Fritz, G., \& Beck, C. 2006 , A\&A, 460, 925

Müller, D. A. N., Schlichenmaier, R., Steiner, O., \& Stix, M. 2002, A\&A, 393, 305

Puschmann, K. G., Kneer, F., Nicklas, H., Wittmann, A. D. 2007, in: Kneer, F., Puschmann, K. G., Wittmann, A.D. (eds.) Modern Solar Facilities - Advanced Solar Science, p. 45

Puschmann, K. G., Kneer, F., Seelemann, T., Wittmann, A. D. 2006, A\&A, 451, 1151

Puschmann, K. G., Ruiz Cobo, B., Vázquez, M., Bonet, J. A., \& Hanslmaier, A. 2005, A\&A, 441, 1157

Puschmann, K. G. \& Sailer, M. 2006, A\&A, 454, 1011

Rempel, M., Schüssler, M., \& Knölker, M. 2009a, ApJ, 691, 640

Rempel, M., Schüssler, M., Cameron, R.H., \& Knölker, M. 2009b, Science, 325, 171

Rimmele, T. \& Marino, J. 2006, ApJ, 646, 593

Ruedi, I., Solanki, S. K., \& Livingston, W. 1995, A\&A, 302, 543

Ruiz Cobo, B. \& Bellot Rubio, L. R. 2008, A\&A, 488, 749 
Ruiz Cobo, B. \& del Toro Iniesta, J. C. 1992, ApJ, 398, 375

Sánchez Almeida, J. 1998, ApJ, 497, 967

Sánchez Almeida, J. 2005, ApJ, 622, 1292

Sánchez Almeida, J., Ruiz Cobo, B., \& del Toro Iniesta, J. C. 1996, A\&A, 314, 295

Sánchez Cuberes, M., Puschmann, K. G., \& Wiehr, E. 2005, A\&A, 440, 345

Scharmer, G. B. 2006, A\&A, 447, 1111

Scharmer, G. B. 2008, Phys. Scr., 133, 014015

Scharmer, G. B. 2009, Space Science Reviews, 144, 229

Scharmer, G. B., Gudiksen, B. V., Kiselman, D., Löfdahl, M. G., \& Rouppe van der Voort, L. H. M. 2002, Nature, Volume 420, Issue 6912 , pp. 151

Scharmer, G. B., Narayan, G., Hillberg, T., et al. 2008, ApJ, 689, L69

Scharmer, G. B. \& Spruit, H. C. 2006, A\&A, 460, 605

Schlichenmaier, R. 2009, Space Science Reviews, 144, 213

Schlichenmaier, R. \& Collados, M. 2002, A\&A, 381, 668

Schlichenmaier, R., Jahn, K., \& Schmidt, H. U. 1998a, A\&A, 337, 897

Schlichenmaier, R., Jahn, K., \& Schmidt, H. U. 1998b, ApJ, 493, 121

Schlichenmaier, R., Müller, D. A. N., Steiner, O., \& Stix, M. 2002 A\&A, 381, L77

Schlichenmaier, R. \& Solanki, S. K. 2003, A\&A, 411, 257

Schmidt, W. \& Fritz, G. 2004, A\&A, 421, 735

Solanki, S. K. 2003, Astronomy and Astrophysics Review, 11, 153

Solanki, S. K. \& Montavon, C. A. P. 1993, A\&A, 275, 283

Solanki, S. K. \& Rüedi, I. 2003, A\&A, 411, 249
Spruit, H. C. \& Scharmer, G. B. 2006, A\&A, 447, 343

Sütterlin, P., Bellot Rubio, L. R., \& Schlichenmaier, R. 2004, A\&A, 424, 1049

Title, A. M., Frank, Z., Shine, R. A., Tarbell, T. D., Topka, K. P., Scharmer, G. \& Schmidt, W. 1993, ApJ, 403, 780

Tritschler, A. 2009, In: Proceedings of the Second Hinode Science Meeting, M. Cheung et al. (eds.), ASP Conf Series, in press, arXiv:0903.1300

Tsuneta, S., Ichimoto, K., Katsukawa, Y., et al. 2008, Sol. Phys., 249,167

Vernazza, J. E., Avrett E. H., \& Loeser, R. 1981, ApJS, 45, 635 (VAL-C)

van Noort, M., Rouppe van der Voort, L., \& Löfdahl, M. G. 2005, Sol. Phys., 228, 191

Volkmer, R., von der Lühe, O., Kneer, F., et al. 2007, in: Kneer, F., Puschmann, K.G., Wittmann, A.D. (eds.) Modern Solar Facilities - Advanced Solar Science, p. 39

Weiss, N. O., Thomas, J. H., Brummell, N. H., \& Tobias, S. M. 2004, ApJ, 600, 1073

Westendorp Plaza, C., del Toro Iniesta, J. C., Ruiz Cobo, B. Martínez Pillet, V., Lites, B. W. \& Skumanich, A. 2001, ApJ, 547,1130

Westendorp Plaza, C., del Toro Iniesta, J. C., Ruiz Cobo, B., \& Martínez Pillet, V. 2001, ApJ, 547, 1148

Wöger, F. \& von der Lühe, O. 2008, Proc. SPIE, 7019, 46

Zakharov, V., Hirzberger, J., Riethmüller, T. L., Solanki, S. K., \& Kobel, P. 2008, A\&A, 488, L17 